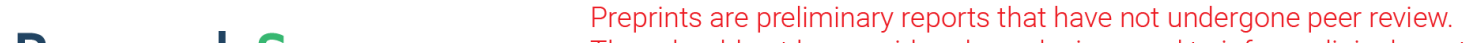 They should not be considered conclusive, used to inform clinical practice, or referenced by the media as validated information. \\ Parallel processing of sensory cue and spatial information in the Dentate Gyrus
}

\section{Sebnem Tuncdemir}

Columbia University

Andres Grosmark

Columbia University

Gergely Turi

Columbia University https://orcid.org/0000-0001-5651-9459

\section{Amei Shank}

Columbia College

John Bowler

Columbia University

\section{Gokhan Ordek}

Columbia University

\section{Attila Losonczy}

Columbia University https://orcid.org/0000-0002-7064-0252

\section{Rene Hen ( $\nabla$ rh95@columbia.edu )}

Columbia University https://orcid.org/0000-0002-7763-4162

\section{Clay Lacefield}

Columbia University

\section{Article}

Keywords: dentate gyrus, DG, cognitive map formation, sensory cue, spatial information, mice, sensory cue response

Posted Date: September 29th, 2020

DOI: https://doi.org/10.21203/rs.3.rs-76845/v1

License: (a) (i) This work is licensed under a Creative Commons Attribution 4.0 International License. Read Full License

Version of Record: A version of this preprint was published at Cell Reports on January 1st, 2022. See the published version at https://doi.org/10.1016/j.celrep.2021.110257. 



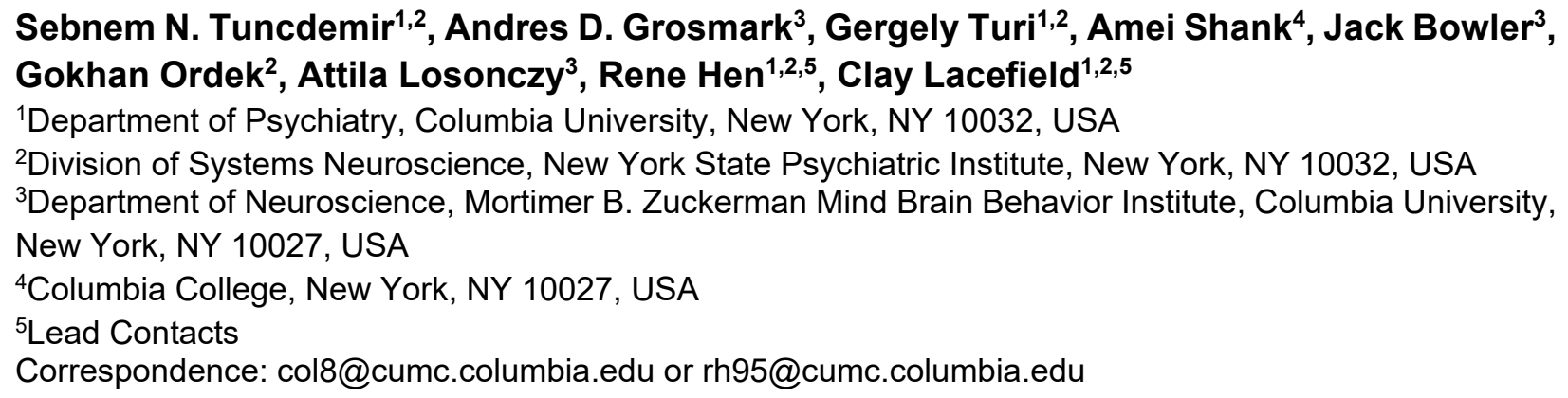

\section{Abstract}

During exploration, animals form an internal map of an environment by combining information about specific sensory cues or landmarks with the animal's motion through space, a process which critically depends on the mammalian hippocampus. The dentate gyrus (DG) is the first stage of the hippocampal trisynaptic circuit where self-motion and sensory cue information are integrated, yet it remains unknown how neurons within the DG encode both cue related ("what") and spatial ("where") information during cognitive map formation. Using two photon calcium imaging in head fixed mice running on a treadmill, along with on-line sensory cue manipulation at specific track locations, we have identified robust sensory cue responses in DG granule cells largely independent of spatial location. Granule cell cue responses are stable for long periods of time, selective for the modality of the stimulus and accompanied by strong inhibition of the firing of other active neurons. At the same time, there is a smaller fraction of neurons whose firing is spatially tuned but insensitive to the presentation of nearby cues, similar to traditional place cells. These results demonstrate the existence of "cue cells" in addition to the better characterized "place cells" in the DG, an important heterogeneity that has been previously overlooked. We hypothesize that the observed diversity of representations within the granule cell population may support parallel processing of complementary sensory and spatial information and impact the role of the dentate gyrus in spatial navigation and episodic memory.

\section{Introduction}

An animal's location in an environment is highly relevant for guiding its behavior, both to find areas of potential reward and avoid areas of possible danger. The mammalian hippocampal formation plays a cardinal role in navigation and spatial memory by integrating self-motion and sensory cue information into a cognitive map of an environment, exemplified by the presence of "place cells" selective for specific locations within space'. As the initial stage in the 'trisynaptic circuit'2, the dentate gyrus (DG) is the first region in the hippocampus to integrate sensory and self-motion information into a discrete spatial representation, and thus represents the most basic state of spatial map formation ${ }^{3,4}$. Yet causal evidence for conjunctive encoding of sensory and spatial information by the principle neurons of the DG, granule cells, is still lacking. While recent studies have shown that granule cells may be activated by visual ${ }^{5}$, tactile ${ }^{6}$ and olfactory ${ }^{7}$ cues, their 
relationship to canonical 'place cells' and their influence on spatial map formation in the DG have not been investigated.

The DG receives its main long-range excitatory inputs from the lateral and medial entorhinal cortices (LEC and MEC, respectively), and sends mossy fiber projections only to area CA $3^{8}$. The LEC is thought to primarily represent information about sensory cues, while the MEC is thought to more prominently encode self-motion information ${ }^{9-11}$. Since individual granule cell dendrites receive projections from both of these areas, these inputs have the potential to be the basis for dendritic computations that combine sensory and self-motion information into a discrete spatial representation ${ }^{12-14}$. In concert with a highly effective winner-take-all process mediated by lateral inhibition ${ }^{15-17}$, these conjunctive representations could facilitate behavioral discrimination of nearby salient locations with high spatial resolution ${ }^{18,19}$. Such processes may underlie proposed computational roles of the DG in hippocampal information processing such as pattern separation, where similar inputs are represented distinctly within the population in order to aid the selectivity of spatial behavior and memory ${ }^{16,20}$.

We sought to examine how the DG participates in spatial map formation by recording calcium activity in large populations of granule cells in the mouse dorsal DG during head-fixed locomotion on a treadmill. By controlling the administration of sensory cues and their pairing with the animal's position on the treadmill, we were able to dissect sensory and spatial contributions to granule cell firing. We found that surprisingly most of the task-associated neurons were highly sensitive to specific sensory cues presented along the treadmill belt, rather than discrete locations. Cue responses in single neurons were stable for long periods of time, selective for the modality of the stimulus and accompanied by strong inhibition of other active neurons. At the same time, a smaller fraction of neurons exhibited robust spatial tuning independent of local cue presentation, yet were more context selective. These two channels of information, sensory and spatial, were largely distinct within the granule cell population, and led us to postulate the existence of "cue cells" in addition to the better characterized "place cells" of the region. This work suggests that the DG maintains a largely parallel code for cues in an environment and their location; yet, possesses specific points of integration, for example through mutual inhibition and the spatial modulation of cue responses. Such properties of the heterogeneous population of cue cells and place cells may play a role in higher level functions of the dentate gyrus such as pattern separation and spatial map formation.

\section{$\underline{\text { Results }}$}

To investigate the interaction between sensory and spatial representations in the dentate gyrus (DG), we used two photon calcium imaging of large populations of granule cells in head-restrained mice running on a treadmill as a virtual linear track ${ }^{21}$. Mice were injected unilaterally with rAAV.Syn.GCaMP6s to express the genetically encoded calcium indicator GCaMP6s in the dorsal DG. This was followed by the implantation of a chronic imaging window above the hippocampal fissure, which allowed us to image the calcium dynamics of neurons in the granule cell layer during treadmill behavior (Fig. 1a). After habituation, mice were trained to run in order to receive randomly delivered water rewards on a $2 \mathrm{~m}$-long treadmill belt, during 15 minute sessions (Fig. $1 \mathrm{~b}$, 
methods). Movies of population calcium imaging data were motion corrected offline ${ }^{22}$, and the activity of putative single neurons was isolated ${ }^{23,24}$. Neurons with significant spatial tuning of calcium activity along the track were identified using previously described methods ${ }^{21,25}$, and in a subset of sessions activity of individual neurons was tracked in multiple conditions over multiple sessions ${ }^{26}$.

In order to isolate the effects of locomotion on hippocampal activity ${ }^{27}$, the treadmill was motorized at a constant speed, adjusted for each mouse (motorized velocity $=10.11 \pm 0.64 \mathrm{~cm} / \mathrm{s}$, self-driven velocity $=12.76 \pm 2.45 \mathrm{~cm} / \mathrm{s}$ ). Despite high velocity dependence of $D G$ activity in freely running mice (Supplementary Fig. 1a), there were no significant differences in the fraction of spatially selective neurons or their mean firing rates in mice running on the motorized treadmill compared to mice advancing the treadmill belt through self-driven locomotion (Supplementary Fig. 1b, c), however mean spatial tuning was higher in mice running on the motorized treadmill (Supplementary Fig. 1d).

\section{Granule Cell Cue Responses}

To investigate the relationship between discrete sensory cues and spatial representations within the DG, we introduced a $1 \mathrm{~s}$ odor pulse delivered in the middle of the track on each lap as a dynamic spatial cue, in addition to a tactile cue at the lap boundary. We found that the majority of spatially selective neurons exhibited receptive fields near the lap boundary and middle locations, corresponding to the lap cue and middle cue positions, respectively (Fig. 1c, $57 \%$ of cells with peak activity within $10 \mathrm{~cm}$ of cues, $43 \%>10 \mathrm{~cm}$ from cues, $p<0.0001$, Mann-Whitney test). Across the population, average firing rates (Fig. 1e) and spatial information (Supplementary Fig. 3a) of spatially tuned neurons were also higher in positions corresponding to sensory cues. These neurons could however be place cells that are enriched at the locations of salient stimuli, as has been shown in area CA1 $1^{28-32}$ or alternately could be directly driven by the stimulus ${ }^{7}$. To test this, the olfactory cue was omitted or shifted $1 / 4^{\text {th }}$ of the track length once every 3-5 trials interleaved throughout the session ( $41 \pm 2$ total laps/session) in order to dissociate cue responses from track location. Under these conditions, a majority of neurons normally active in the middle of the track shifted their firing position to match the new location of the odor in cue-shift trials (Fig. 1d, middle, $79.07 \pm 2.76 \%$ ) and exhibited reduced activity in cue-omitted trials (Fig. 1d, right, $78.2 \pm 3.87$ $\%$ ), compared with normal middle cue laps ( $n=285$ spatially tuned neurons from 8 mice). In contrast, neurons firing at locations corresponding to the invariant lap cue were unchanged in omit and shift laps. On trials with a shifted middle cue, average firing rates were higher within the new cue region (red, inset $p=0.0234$ Signed Rank test), and lower at the normal cue location on cue-omitted trials (blue, inset $p<0.00001$, Signed Rank Sum test) compared to the corresponding positions during normal trials (Fig. 1e, inset). Thus, a substantial population of spatially tuned DG neurons in the virtual linear track environment are in fact active directly in response to presentation of cues at those locations, rather than the locations themselves.

Comparable population responses were found in response to cues of other sensory modalities, such as visual or tactile cues (Supplementary Fig. 2a-d), as well for liquid rewards (Supplementary Fig. 2e-h). In addition, increasing the complexity of the environment with two additional cues at 
other track locations results in an additive pattern of single cue responses (Supplementary Fig. $2 \mathrm{i}-\mathrm{k}$ ), suggesting that strong cue responses are not limited to situations with a single cue alone. Cue responses were also similar for cells imaged in the dorsal DG of a granule cell-specific Cre transgenic mouse (Dock10 $\mathrm{Cre}^{33}$, Supplementary Fig. 3b-d,), indicating that sensory cue representations are indeed a property of the granule cell population rather than arising from other local neuron types such as mossy cells or inhibitory interneurons ${ }^{6}$.

Next, we divided the spatially tuned population of granule cells into three groups for subsequent analyses based on the position of their spatial fields and their activity during cue manipulation (omit or shift) trials. The three response types are illustrated for one session (Fig. 1f): 1) cells with spatial fields within the middle cue region that closely track the changes in cue presentation ("odor-cue cells", top); 2) cells with spatial fields around the lap boundary cue on the treadmill belt ("lap-cue cells", middle); and 3) the remaining spatially tuned cells with receptive fields outside of the cue locations throughout the track ("place cells", bottom). These three groups, odor-cue, lapcue, and place cells, constituted $22.5 \pm 2 \%, 47.1 \pm 2 \%$ and $30.4 \pm 1 \%$ of the spatially tuned cells within the imaging field of view, respectively (Supplementary Fig. 3e). We found that cells with similar response types did not cluster together spatially within the imaging field and the groups did not exhibit significant differences in overall mean firing rates (Supplementary Fig. 3e-g). Spatial coding properties however differed between cue and place coding populations of neurons. Both populations of cue cells (lap cue and olfactory cue) showed higher average spatial information (Fig. 1g, $X^{2}=48.47, p<0.0001$, Kruskal-Wallis test) and had more consistent spatial firing between the first and the second half of each session than place cells (Fig. $1 \mathrm{~h}, X^{2}=32.60$, $p<0.0001$, Kruskal-Wallis test).

In order to probe the emergence of selective firing in these populations, we identified the lap in which responses began to robustly occur within the preferred spatial location during the first session of exposure to the odor cue (field onset lap ${ }^{34}$ ). We found that for odor and lap cue cells respectively) while the majority of place cells emerged later in the session $(52 / 84(62 \%)$ within 10 laps, Fig. $1 i, x^{2}=9.29, p=0.0096$, Kruskal-Wallis test). In agreement with the later emergence of place cells, we found that the in-field firing rates of place cells were significantly smaller than those of odor and lap cue cells only within the first 5 laps (Fig. 1i, inset, 2-way ANOVA, main effect of cell type: $F_{2,1473}=6.73, p=0.0012$; cell type $\times$ lap number interaction $F_{4,1473}=2.44, p=0.04$; main effect of lap number: $F_{2,1473}=0.28, p=0.7$ ). Additional experiments also showed that on an uncued treadmill belt prior to olfactory cue sessions, the majority of 'future' odor cue cells were either inactive or had low spatial tuning (Supplementary Fig. 4), suggesting that cue cells rapidly emerge de novo and are not place cells that remap to new cue locations. Taken together, these results demonstrate that sensory cue representations are more reliable and appear with less exposure than place cell representations, and arise from an independent population of granule cells.

\section{Stability and Specificity of Sensory Cue Responses}

To further characterize the stability and coding specificity among the granule cell subpopulations, we investigated the responses of individual neurons over time and with respect to different 
sensory cues (Fig. 2a, b). We utilized an analytical method ${ }^{26}$ to track cells over multiple sessions and were able to find substantial numbers of the same cells active in different sessions within a day or 1 week later in the same fields of view (Supplementary Fig. 5a, b). Although not all cells were identified in every session, a similar percentage of spatially selective neurons was registered in all sessions, which was confirmed by visual inspection to ensure that cells appeared consistent in the anatomical images (Supplementary Fig. 5c-g).

Between any two sessions, over days or with different cue modalities, the cells encoding the invariant lap cue were the largest fraction of cells that remained active and maintained their response type (e.g. cue type and/or place, green circles in Fig. 2c). Odor cue cells fired reliably to the same olfactory cue over long periods of time (blue circles in red and blue shaded area, Fig. 2c), but were largely unresponsive to cues of other modalities presented at the same position (green shaded area in Fig. 2c, $x^{2}=19.78, p<0.0001$, Kruskal-Wallis test). Conversely, a lower percentage of place cells maintained their response type across days compared to cue cells recorded in the same sessions using the same cues (orange circles in blue shaded area, Fig. 2c, $X^{2}=14.36, p<0.001$, Kruskal-Wallis test). Furthermore when we examined specifically whether cue cells become place cells between these sessions, or vice versa, we found that categorical cue and place representations remain extremely stable within the DG population (Fig. 2d, $p<0.001$, Rank Sum test).

We further examined the degree of stability and specificity of cue and place cell tuning by crosscorrelating spatial firing rates for individual registered cells over time and with respect to different sensory cues (Fig. 2e). For this analysis, we computed the correlation of spatial firing rates for cells registered during different sessions within a day and one week later, or with a different sensory cue modality. In line with the stimulus selectivity analysis described above, we observed that middle location odor cue cells displayed significantly lower correlations in sessions with a different sensory cue, compared to separate sessions using the same olfactory cue on the same day or one week later (left, $x^{2}=27.88, p<0.0001$, Kruskal Wallis test). Thus, cells that responded to a cue of one modality were unlikely to respond to cues of other modalities, despite a similar spatial location of the cue between sessions. Correlations in the activity of individual place cells over $1 \mathrm{wk}$. were significantly lower than on the same day, and were lower than both cue cell populations, again indicating lower stability for place than cue representations (left, $X^{2}=18.79$, $p<0.0001$, Kruskal Wallis test). Lap cue cells did not display significant changes in their firing rate correlations between sessions on the same day, across days or with different sensory cues, and were therefore especially stable.

Responses of individual odor cue cells were also highly correlated in sessions recorded in different rooms on the same day (Fig. 2f-h), indicating that distal ambient cues have a limited influence on the stability of sensory cue representation in DG granule cells. Despite the high stability of cue cells, place cells were significantly less consistent between rooms than cue cells in the same sessions (Fig. $2 \mathrm{~g}, \mathrm{~h}, \mathrm{p}<0.001$, Kruskal-Wallis test), and were similar to that seen in place cells over $1 \mathrm{wk}$ when measured in the same room (Fig. 2e), suggesting that place cells are more context selective than cue cells. Taken together, these results suggest that sensory cues are represented by a stable subpopulation of neurons that is highly selective for specific cues 
while purely spatial representations are less stable and undergo progressive reorganization over time and in different global contexts.

\section{Spatial Modulation of DG Cue Responses}

The juxtaposition of inputs from the lateral and medial entorhinal cortex onto the dendrites of individual granule cells has been hypothesized to underlie a conjunctive code for sensory cues and their spatial location ${ }^{3,4}$. We therefore examined the influence of spatial location on cue responses ("spatial modulation") by tracking odor cue cells through multiple sessions with different cue-location pairing, either with an intermittently shifted cue as in previous experiments or in separate sessions with the same cue at random locations each lap (Fig. 3a, b). On average, cuetriggered $\mathrm{Ca}^{2+}$ response amplitudes for individual cue cells (a proxy for neuron action potential burst firing rate) were smaller when cues were presented at the infrequent "shift" location or at random locations, when compared to their responses at the more frequent middle location (Fig. $3 c, d, p<0.0001$, Friedman test, $n=101,5$ mice). Thus while cue cells tend to respond to the same sensory cue regardless of location, the strongest responses occur when a cue is presented at the same place repeatedly.

To further examine the spatial modulation of cue responses, we performed a set of experiments where a visual cue was delivered at multiple locations on the track to measure selectivity of granule cell responses to cues presented consistently at distinct locations ("dual location cue", Fig. 3e), similar to recent work by other groups using virtual reality environments ${ }^{35,36}$. As in our previous experiments with a single cue, each cue was omitted on a subset of laps to isolate cue cells as opposed to place cells present in the vicinity of cues (Supplementary Fig. 6a, b). To measure the extent of preference for one cue location versus the other, we calculated the spatial modulation index for each cue cell as the ratio of the firing rates in the non-preferred cue location versus the preferred cue location. Cue cells in the first session exhibited a range of spatial modulation indices, but over the population their event rates were significantly modulated by the cue location $(p<0.00001$, Wilcoxon sign rank test on day 1 spatial modulation index, $53 \%$ of individual neurons significantly spatially modulated by location shuffle). Spatial modulation also increased over several days of dual cue presentations ( $p=0.0125$ Wilcoxon ranked sum test, day 1 vs 2,3 , or 4 ), with $81 \%$ of cue cells exhibiting significant spatial modulation by day 4 (Fig. $3 g, h$ and Supplementary Fig. 6c, d). This suggests that dentate cue cells are acutely modulated by the spatial location of two identical cues but increase their preference for a single location over days.

\section{Effects of Cues on Spatial Encoding in the DG}

While the most robust activity in the DG was found within the cue cell population, the existence of the smaller population of place cells in uncued locations suggests that spatial activity in these cells is referenced to one of the two cues on the otherwise featureless treadmill track in order to encode a unique location. We therefore examined the effect of manipulations of the variable cue on the subsequent spatial encoding of place cells, in order to judge the degree to which this cue acts as a landmark. For example, if place cells were acutely referenced to the nearest cue we would expect the place fields of cells following the variable middle cue to shift on cue shifted laps. 
To examine the relationship between spatial firing patterns in normal cue laps to those in cue shifted laps, we first calculated population vector (PV) correlations of firing rates across all spatially tuned cells on each lap (Fig. 4a and Supplementary Fig. 7a). While the correlation was higher in the vicinity of the cues between normal laps, we observed a dramatic decrease in PV correlation between normal and cue shifted laps which was confined to the area immediately To further evaluate the manner in which cue associated activity contributes to spatial encoding, a spatial Bayesian decoder was constructed from the firing rate vectors of all spatially tuned cells $(4,091 \text { cells from } 66 \text { sessions })^{25}$. In order to establish a non-biased estimate of the position during treadmill running, the decoding was performed using a 5 fold cross validation approach in which the $1 / 5^{\text {th }}$ of decoded laps were held out from the training set. Post-reconstruction, we divided the data according to the lap types (Fig. $4 \mathrm{~b}$ and Supplementary Fig. 7b, c). The decoder accuracy was higher in normal (median, $10.6 \mathrm{~cm}$ ) compared to both cue shift (median, $19.6 \mathrm{~cm}$ ) and cue omitted laps (median, $18.4 \mathrm{~cm}, \mathrm{p}<0.0001$, Wilcoxon rank sum test, Fig. $4 \mathrm{c}$ and Supplementary Fig. $7 \mathrm{~d}$, e), indicating that cue manipulations affect the accuracy of spatial coding by the DG population. However, while spatial decoding was initially strongly perturbed by the cue shift, the decoder error on shift laps soon converged to that of normal laps, well before the subsequent lap cue (Fig. 4d, dotted line). Together these results suggest that as a population, the spatial encoding of granule cells is affected by local cues for a limited time, rather than persistently altering their estimate of the animal's position.

Population vector correlations and Bayesian decoding are average measures of spatial responses for the granule cell population as a whole, which might obscure differences in how the activity of individual cells is referenced to local or global cues to encode an animal's location. We therefore examined responses of individual granule cells by measuring correlations between their spatial firing in normal and cue-shifted laps. In doing so we found two general classes of single-cell responses: some cells were acutely affected by the intermittently shifting odor cue, while others were referenced to the stable lap boundary cue (Fig. 4e, f and Supplementary Fig. 7f, g). The majority of cells that shifted their firing in reference to the new cue location $(0.5 \mathrm{~m})$ were normally active immediately in response to the middle cue ("cue-influenced"), with a smaller number persistently affected by the shift prior to the next lap cue. In contrast, a relatively constant number of tuned cells along the track maintained their normal firing location with reference to the distant lap boundary cue despite the shifted local cue ("non cue-influenced"). Notably, there was a small enrichment of such place cells immediately prior to the cue but unaffected by the cue shift, indicating a spatial response predictive of salient cues. These data suggest that the DG population maintains independent spatial reference frames based upon distinct local and global cues within an environment. This code however differs depending upon the spatial stability of the cues: the major effects of a variable cue presentation are limited to cells that fire directly in response to the cue, while a majority of place cells over the track are preferentially influenced by the stable cue as a landmark. 

Spatial receptive fields of granule cells are hypothesized to form within a competitive network in which lateral inhibition mediated by GABAergic interneurons enforces sparse encoding that may aid pattern separation ${ }^{15}$. The robust and highly selective cue responses of granule cells in our behavioral paradigm allowed us to examine patterns of local-circuit inhibition within the DG network. First, we sought to analyze the effect of cue responses on nonselective background firing observed within the spatially tuned granule cell population. By comparing out-of-field firing rates at the middle cue location in normal laps with laps where the cue is omitted, we identified a significant reduction in background firing of the granule cell population during the cue presentation (Fig. 5a, p=0.0004, Signed Rank Sum test). This suppression was absent on cue-omitted laps and generally co-varied with the mean amplitude of cue-related excitation on a session-bysession basis (Fig. 5b, $R=0.22, p<0.0001$, Signed Rank Sum test). Furthermore, the timing of the peak of this inhibition was delayed with respect to the excitatory cue response in these sessions (Supplementary Fig. 8a, b). These data show that sensory cue input is able to suppress 'noisy', nonspecific activity in the vicinity of the cue, potentially through lateral inhibition.

In addition to suppressing nonspecific activity in the DG network, we sought to determine the effect of cue responses on activity in place cells and in cells responding to other cues. By examining place cells with receptive fields normally at the shift location, we found that firing rates were strongly suppressed on shift laps when a cue was presented at this location compared to normal laps when a cue was not present here (Fig. 5d, e). Furthermore when two distinct cues were normally presented at different locations, shifting of the position of one cue to the other cue location typically had the effect of suppressing the responses in both cue populations (Fig. 5f, g), indicating mutual inhibition among granule cells encoding different cues. Thus in addition to the inhibition of nonspecific firing, cue responses lead to overall inhibition of place cell responses, and responses to competing cues as well. Taken together, our results suggest that cue-related activity in granule cells results in strong suppression of responses in neighboring cells via lateral inhibition.

\section{Discussion}

By imaging calcium activity in large populations of dentate granule cells during head-fixed spatial behaviors on a virtual linear track, we have shown that a major population of task-selective neurons is highly sensitive to specific sensory cues rather than to discrete locations. The ability to dynamically manipulate a sensory cue and its association with locations on the treadmill track allowed us to isolate the population of cue cells, in addition to the complementary population of spatially tuned place cells recorded in the same sessions.

Properties of cells within these groups also differed: sensory cue responses were highly-tuned and remarkably robust across contexts and over time- significantly more so than the responses of the canonical place cell population. And while cue cell responses were not present initially, they emerged rapidly during the first exposures to a cue while place cell responses emerged more slowly. These findings suggest that dentate cue cells stably represent basic features of sensory 
cues in an environment, while place cells constitute a more dynamic population that gradually adapts to current conditions, for example, by integrating between stable cues in order to provide an accurate estimate of the animal's location when no cues are present ${ }^{37}$.

\section{Heterogeneous properties of Granule Cells}

The robust and consistent nature of GC cue responses suggests that cue-selective populations may have been similarly present in previous experiments recording DG activity in vivo, however the lack of precise stimulus control made it impossible to distinguish cue-responsive versus placeresponsive components $5,6,38$. Indeed, spatial receptive fields of individual granule cells measured in different environments with similar sensory cues display mixed levels of changes in their firing rates, with a subset of granule cells completely remapping their firing fields while others show more stable dynamics ${ }^{5,38-41}$. Based upon our results, these subgroups may correspond to place cells and cue cells, respectively. A major impact of sensory cues on DG firing largely independent of spatial context might also explain the relatively lower context selectivity observed in the DG compared with other hippocampal subfields, at least when measured in different contexts that contain the same or similar sensory cues ${ }^{5,38-41}$. To support this notion, we found that DG cue cells were largely stable when recorded over long periods of time, with different cue positions, or in different room contexts, while place cells were not.

Previous work has demonstrated that the two major long range inputs to the dentate gyrus, the lateral and medial entorhinal cortex (LEC and MEC), are involved with processing functionally distinct information 9,10 . This raises the possibility that our "cue cells" may be driven primarily by sensory inputs from the LEC, while "place cells" are driven by self-motion information relayed from the MEC, for example in the form of grid cell responses. The segregation of these properties within the overall granule cell population suggests that these streams of information remain largely separated at the level of the DG, similar to the functional heterogeneity observed in other hippocampal subfields ${ }^{42,44}$. In these regions such heterogeneity among principal neurons has been hypothesized to be critical for efficient encoding of spatial representations within a local population ${ }^{42-44}$. Heterogeneity has also been observed in immediate early gene expression profiles within DG neurons encoding individual memory engrams ${ }^{45}$ which are differentially targeted by entorhinal afferent pathways ${ }^{8,45-47}$. These findings are in line with our study demonstrating that sensory and spatial information remain partially separate at the level of the DG, and suggest that multiple channels of spatial and non-spatial information contribute distinctly to local and down-stream computations. This separation is also consistent with current ideas that cue-based and path-integration based navigation are complementary, rather than integrated, in order to produce place-specific firing both in locations where landmark cues are present as well as in between cues ${ }^{48}$.

Furthermore, the separation of cue and place responses within the granule cell population suggests that specific inputs from either LEC or MEC dominate individual granule cell firing. This might result from the unique cellular properties of granule cells, local circuits such as inhibitory networks ${ }^{49}$, as well as the laminated pattern of LEC and MEC inputs onto granule cell dendrites ${ }^{8}$. Inputs from distinct cortical afferents can be strongly filtered due to hyperpolarized resting 
membrane potentials and substantial attenuation of regenerative dendritic activity in granule cells $^{12,13}$. Furthermore, short coincidence-detection windows for EPSPs ${ }^{50}$ may result in a high activation threshold of each cortical input. Such gating of inputs by postsynaptic neuronal excitability may provide a cellular substrate for our observation that these channels of information remain largely separated at the level of the DG. Differences in input sources may also explain the distinct time course of emergence of cue and place cell responses, which could reflect divergent mechanisms for synaptic plasticity between LEC and MEC inputs, differential inhibition of these pathways, or other factors such as associative vs. non-associative learning. Future studies investigating whether cue and place encoding populations receive different inputs from LEC and MEC, as well as the molecular basis of the heterogeneity among granule cell populations, will allow for a more detailed inquiry into the functional diversity in DG granule cells. Such studies may also allow us to selectively target cue and place cells in order to manipulate their activity and determine their roles during discrimination behaviors.

\section{Functional significance of dentate gyrus cue cell properties}

But how do these results inform our ideas about how the DG participates in pattern separation and spatial map formation? First, we found that although individual granule cells respond to the same cues when presented in different locations, the strength of this activity was spatially modulated. Differential encoding of cues based upon their spatial location may contribute to pattern separation by differentiating between similar cues present in different places within an environment, or in distinct contexts. We observed that spatially modulated cue responses also increased over days when a single cue was repeatedly presented at multiple locations, indicating plasticity in location specificity and potentially progressive enhancement of pattern separation. Notably, responses were also on average larger for odor cues presented at a single location, as opposed to shifted or random locations. This suggests that cue responses are more robust for stimuli paired repeatedly with one particular location, which may indicate dendritic integration of sensory and spatial information. Conjunctive encoding of sensory cues and their locations within an environment could play a role in establishing landmarks for spatial navigation. In support of this idea, we found that most place cells were referenced to the stable lap cue rather than the variable middle cue, indicating that this cue is preferentially utilized as a landmark.

Our study also shows that cue responses lead to potent inhibition of three distinct types of granule cell activity: nonspecific "noisy" spiking activity, the spatial firing of place cells normally active in a location, and the activity of cells responsive to other distinct sensory cues. This supports the hypothesis of a competitive network in the DG enforced by strong inhibition, which has been suggested to contribute to pattern separation. In addition, suppression of weaker place cell responses by cue-related activity may indicate that the network is organized to utilize the strongest and perhaps the most informative parameters, such as landmark cues, in order to establish an animal's location in space.

440

Furthermore, rate modulation of granule cell cue responses resulting from spatial location or association with other cues is itself a form of "rate remapping", a feature frequently attributed to the dentate gyrus in pattern separation. Thus spatial location, as well as the juxtaposition of 
multiple cues and their associated patterns of inhibition, may create a rate modulated landscape of granule cell activity specific to the current context. The overall pattern of contextually modulated cue responses in the DG may form the basis for recruitment of distinct populations of neurons, or "global remapping", observed in area CA3. Slower emergence and lower stability over sessions in the place cell population may also point to a role for these neurons in context selectivity. Indeed place cells differed more greatly than cue cells recorded under similar conditions in different rooms.

The properties of cue and place cells in the dentate gyrus have the hallmark of features one might expect from the first stage of spatial map formation in the hippocampus: mostly distinct but slightly mixed encoding of sensory cues, or "what", and place, or "where". While place cells in the DG are referenced to stable spatial cues in order to determine a unique location of firing given movementdependent grid cell inputs from MEC, cue cells are mostly driven by sensory input from the LEC, but modulated by the spatial context of the cues. Such complementary channels of information may be further refined by downstream hippocampal areas into an integrated spatial map that encodes relationships between important features such as spatial cues and goals, which can be used to organize adaptive behavior. Uncovering the functional heterogeneity of cue and place cells in the dentate gyrus helps us to better understand the basic neural mechanisms underlying our sense of orientation in complex environments by utilizing both landmarks and self-motion information to guide our movements through space.

\section{Acknowledgements}

We would like to thank Jack Berry for histological photos and other members of the Hen lab for critical insight throughout this project. This work was funded by NIMH T32 MH015144 (S.N.T.), NYSTEM-C029157 (G.O., G.T., C.O.L, R.H.), NIH S10 OD018464, Revson Senior Fellowship in Biomedical Science (A.D.G.), NIMH R01 AG043688 (R.H.), MH068542 (R.H.), NIMH R01 MH100631 (A.L.), NINDS R01NS094668 (A.L.), and NINDS U19NS104590 (A.L.).

\section{Author Contributions}

Conceptualization, C.O.L., S.N.T., and R.H.; Methodology, C.O.L., S.N.T., A.S.; Software, C.O.L., S.N.T., A.D.G., J.B.; Formal Analysis, S.N.T., C.O.L.; Investigation, S.N.T, C.O.L.; Resources, C.O.L., A.D.G., G.T., J.B., A.L., G.O.; Writing, Review and Editing, S.N.T., C.O.L., R.H., A.D.G., A.L.; Supervision, R.H and A.L.

\section{Declaration of Interests}

The authors declare no competing interests.

\section{References}

1. O'Keefe, J. \& Dostrovsky, J. The hippocampus as a spatial map. Preliminary evidence from unit activity in the freely-moving rat. Brain Res. 34, 171-175 (1971).

2. Amaral, D. G., Scharfman, H. E. \& Lavenex, P. The dentate gyrus: fundamental neuroanatomical organization (dentate gyrus for dummies). Prog. Brain Res. 163, 3-22 (2007).

3. Kesner, R. P. An analysis of the dentate gyrus function. Behavioural Brain Research 254, $1-7$ (2013). 
486

487

488

489

490

491

492

493

494

495

496

497

498

499

500

501

502

503

504

505

506

507

508

509

510

511

512

513

514

515

516

517

518

519

520

521

522

523

524

525

526

527

528

529

530

531

532

533

534
4. Lee, J. W. \& Jung, M. W. Separation or binding? Role of the dentate gyrus in hippocampal mnemonic processing. Neuroscience \& Biobehavioral Reviews 75, 183-194 (2017).

5. Hainmueller, T. \& Bartos, M. Parallel emergence of stable and dynamic memory engrams in the hippocampus. Nature 558, 292-296 (2018).

6. Jung, D. et al. Dentate granule and mossy cells exhibit distinct spatiotemporal responses to local change in a one-dimensional landscape of visual-tactile cues. Sci Rep 9, 9545 (2019).

7. Woods, N. I. et al. The Dentate Gyrus Classifies Cortical Representations of Learned Stimuli. Neuron 107, 173-184.e6 (2020).

8. Witter, M. P. The perforant path: projections from the entorhinal cortex to the dentate gyrus. Prog. Brain Res. 163, 43-61 (2007).

9. Hargreaves, E. L., Rao, G., Lee, I. \& Knierim, J. J. Major dissociation between medial and lateral entorhinal input to dorsal hippocampus. Science 308, 1792-1794 (2005).

10. Knierim, J. J., Neunuebel, J. P. \& Deshmukh, S. S. Functional correlates of the lateral and medial entorhinal cortex: objects, path integration and local-global reference frames. Philos. Trans. R. Soc. Lond., B, Biol. Sci. 369, 20130369 (2014).

11. Wang, C. et al. Egocentric coding of external items in the lateral entorhinal cortex. Science 362, 945-949 (2018).

12. Kim, S., Kim, Y., Lee, S.-H. \& Ho, W.-K. Dendritic spikes in hippocampal granule cells are necessary for long-term potentiation at the perforant path synapse. Elife 7, (2018).

13. Krueppel, R., Remy, S. \& Beck, H. Dendritic integration in hippocampal dentate granule cells. Neuron 71, 512-528 (2011).

14. McNaughton, B. L., Douglas, R. M. \& Goddard, G. V. Synaptic enhancement in fascia dentata: Cooperativity among coactive afferents. Brain Research 157, 277-293 (1978).

15. de Almeida, L., Idiart, M. \& Lisman, J. E. The input-output transformation of the hippocampal granule cells: from grid cells to place fields. J. Neurosci. 29, 7504-7512 (2009).

16. Marr, D. Simple memory: a theory for archicortex. Philos. Trans. R. Soc. Lond., B, Biol. Sci. 262, 23-81 (1971).

17. Treves, A. \& Rolls, E. T. Computational analysis of the role of the hippocampus in memory. Hippocampus 4, 374-391 (1994).

18. Hunsaker, M. R., Rosenberg, J. S. \& Kesner, R. P. The role of the dentate gyrus, CA3a,b, and CA3c for detecting spatial and environmental novelty. Hippocampus 18, 1064-1073 (2008).

19. Xavier, G. F. \& Costa, V. C. I. Dentate gyrus and spatial behaviour. Prog. Neuropsychopharmacol. Biol. Psychiatry 33, 762-773 (2009).

20. Knierim, J. J. \& Neunuebel, J. P. Tracking the flow of hippocampal computation: Pattern separation, pattern completion, and attractor dynamics. Neurobiology of Learning and Memory 129, 38-49 (2016).

21. Danielson, N. B. et al. Distinct Contribution of Adult-Born Hippocampal Granule Cells to Context Encoding. Neuron 90, 101-112 (2016).

22. Pnevmatikakis, E. A. \& Giovannucci, A. NoRMCorre: An online algorithm for piecewise rigid motion correction of calcium imaging data. J. Neurosci. Methods 291, 83-94 (2017).

23. Giovannucci, A. et al. CalmAn an open source tool for scalable calcium imaging data analysis. Elife 8, (2019).

24. Pachitariu, M. et al. Suite2p: beyond 10,000 neurons with standard two-photon microscopy. bioRxiv 061507 (2016) doi:10.1101/061507.

25. Grosmark, A. D. \& Buzsáki, G. Diversity in neural firing dynamics supports both rigid and learned hippocampal sequences. Science 351, 1440-1443 (2016).

26. Sheintuch, L. et al. Tracking the Same Neurons across Multiple Days in Ca2+ Imaging Data. Cell Rep 21, 1102-1115 (2017). 
535

536

537

538

539

540

541

542

543

544

545

546

547

548

549

550

551

552

553

554

555

556

557

558

559

560

561

562

563

564

565

566

567

568

569

570

571

572

573

574

575

576

577

578

579

580

581

582

583

584

27. Fuhrmann, F. et al. Locomotion, Theta Oscillations, and the Speed-Correlated Firing of Hippocampal Neurons Are Controlled by a Medial Septal Glutamatergic Circuit. Neuron 86, 1253-1264 (2015).

28. Bourboulou, R. et al. Dynamic control of hippocampal spatial coding resolution by local visual cues. eLife 8, e44487 (2019).

29. Hetherington, P. A. \& Shapiro, M. L. Hippocampal place fields are altered by the removal of single visual cues in a distance-dependent manner. Behav. Neurosci. 111, 20-34 (1997).

30. Hollup, S. A., Molden, S., Donnett, J. G., Moser, M. B. \& Moser, E. I. Accumulation of hippocampal place fields at the goal location in an annular watermaze task. J. Neurosci. 21, 1635-1644 (2001).

31. O'Keefe, J. \& Conway, D. H. Hippocampal place units in the freely moving rat: why they fire where they fire. Exp Brain Res 31, 573-590 (1978).

32. Wiener, S. I., Paul, C. A. \& Eichenbaum, H. Spatial and behavioral correlates of hippocampal neuronal activity. J. Neurosci. 9, 2737-2763 (1989).

33. Kohara, K. et al. Cell type-specific genetic and optogenetic tools reveal novel hippocampal CA2 circuits. Nat Neurosci 17, 269-279 (2014).

34. Sheffield, M. E. J., Adoff, M. D. \& Dombeck, D. A. Increased Prevalence of Calcium Transients across the Dendritic Arbor during Place Field Formation. Neuron 96, 490-504.e5 (2017).

35. Saleem, A. B., Diamanti, E. M., Fournier, J., Harris, K. D. \& Carandini, M. Coherent encoding of subjective spatial position in visual cortex and hippocampus. Nature 562, 124127 (2018).

36. Zhao, X., Wang, Y., Spruston, N. \& Magee, J. C. Membrane potential dynamics underlying context-dependent sensory responses in the hippocampus. Nature Neuroscience 23, 881891 (2020).

37. Draht, F. et al. Experience-Dependency of Reliance on Local Visual and Idiothetic Cues for Spatial Representations Created in the Absence of Distal Information. Front Behav Neurosci 11, 92 (2017).

38. Leutgeb, J. K., Leutgeb, S., Moser, M.-B. \& Moser, E. I. Pattern separation in the dentate gyrus and CA3 of the hippocampus. Science 315, 961-966 (2007).

39. Danielson, N. B. et al. In Vivo Imaging of Dentate Gyrus Mossy Cells in Behaving Mice. Neuron 93, 552-559.e4 (2017).

40. GoodSmith, D. et al. Spatial Representations of Granule Cells and Mossy Cells of the Dentate Gyrus. Neuron 93, 677-690.e5 (2017).

41. Senzai, Y. \& Buzsáki, G. Physiological Properties and Behavioral Correlates of Hippocampal Granule Cells and Mossy Cells. Neuron 93, 691-704.e5 (2017).

42. Cembrowski, M. S. \& Spruston, N. Heterogeneity within classical cell types is the rule: lessons from hippocampal pyramidal neurons. Nat. Rev. Neurosci. 20, 193-204 (2019).

43. Chelaru, M. I. \& Dragoi, V. Efficient coding in heterogeneous neuronal populations. Proc. Natl. Acad. Sci. U.S.A. 105, 16344-16349 (2008).

44. Soltesz, I. \& Losonczy, A. CA1 pyramidal cell diversity enabling parallel information processing in the hippocampus. Nat. Neurosci. 21, 484-493 (2018).

45. Sun, X. et al. Functionally Distinct Neuronal Ensembles within the Memory Engram. Cell 181, 410-423.e17 (2020).

46. Luna, V. M. et al. Adult-born hippocampal neurons bidirectionally modulate entorhinal inputs into the dentate gyrus. Science 364, 578-583 (2019).

47. Erwin, S. R. et al. A Sparse, Spatially Biased Subtype of Mature Granule Cell Dominates Recruitment in Hippocampal-Associated Behaviors. Cell Rep 31, 107551 (2020).

48. Poucet, B. et al. Independence of landmark and self-motion-guided navigation: a different role for grid cells. Philos. Trans. R. Soc. Lond., B, Biol. Sci. 369, 20130370 (2014). 
585

586

587

588

589

590

591

592

593

594

595

596

597

598

599

600

601

602

603

604

605

606

607

608

609

610

611

612

613

614

615

616

617

618

619

620

621

622

623

624

625

626

627

628

629

49. Pernía-Andrade, A. J. \& Jonas, P. Theta-gamma-modulated synaptic currents in hippocampal granule cells in vivo define a mechanism for network oscillations. Neuron 81, 140-152 (2014).

50. Schmidt-Hieber, C., Jonas, P. \& Bischofberger, J. Subthreshold dendritic signal processing and coincidence detection in dentate gyrus granule cells. J. Neurosci. 27, 8430-8441 (2007).

51. Skaggs, W. E., McNaughton, B. L. \& Gothard, K. M. An Information-Theoretic Approach to Deciphering the Hippocampal Code. in Advances in Neural Information Processing Systems 5 (eds. Hanson, S. J., Cowan, J. D. \& Giles, C. L.) 1030-1037 (Morgan-Kaufmann, 1993).

\section{Experimental Procedures}

\section{Mice}

All procedures were conducted in accordance with the U.S. NIH Guide for the Care and Use of Laboratory Animals and the Institutional Animal Care and Use Committees of the New York State Psychiatric Institute and Columbia University. Adult male C57BL/6J mice were supplied by Jackson Laboratory and Dock10 cre mice were a gift from Susumu Tonegawa (Massachusetts Institute of Technology). Mice were housed in a vivarium grouped 2-4 mice/cage enriched with running wheels, maintained on a 12-hour light cycle and used at 8-10 weeks of age. Experiments were conducted during the light phase of the cycle. Food and water were available ad libitum until the beginning of the experiment, when they were placed under controlled water supply and maintained at $>90 \%$ of their pre-deprivation weight over the course of imaging experiments. In total, imaging data from 18 mice were used in this study.

\section{Surgery}

Dentate gyrus virus injection and imaging window implantation surgeries were performed as described previously (Danielson 2016, 2017). For all surgical procedures, mice were anesthetized with $1.5 \%$ isoflurane at an oxygen flow rate of $1 \mathrm{~L} / \mathrm{min}$, and head-fixed in a stereotactic frame (Kopf Instruments, Tujunga, CA). Eyes were lubricated with an ophthalmic ointment, and body temperature maintained at $37^{\circ} \mathrm{C}$ with a warm water recirculator (Stryker, Kalamazoo, MI). The fur was shaved and incision site sterilized prior to beginning surgical procedures, and subcutaneous saline and carpofen were provided peri-operatively and for 3 days post-operatively to prevent dehydration and for analgesia. Mice were unilaterally injected with recombinant adeno-associated virus (rAVV) carrying the GCaMP6s transgene (pAAV.Syn.GCaMP6s.WPRE.SV40), purchased

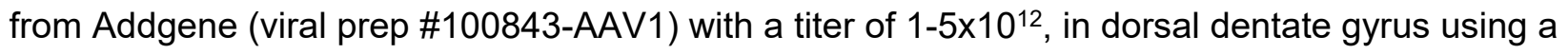
Nanoject syringe (Drummond Scientific, Broomall, PA). Injection coordinates were $-1.5 \mathrm{~mm}$ AP, $-2 \mathrm{~mm} \mathrm{ML}$ from the bregma skull suture, and $-1.85,-1.7,-1.55 \mathrm{~mm}$ DV relative to the cortical surface. $30 \mathrm{~nL}$ of diluted virus was injected at each DV location in $10 \mathrm{~nL}$ increments. Mice were allowed to recover for 3 days and then were implanted with an imaging window over the injected area and stainless steel head-post for head fixation. Imaging windows were constructed by adhering $1.8 \mathrm{~mm}$ diameter, $2.3 \mathrm{~mm}$ long stainless steel hypodermic tubing (Ziggy's Tubes and Wires Inc, Pleasant Hill, TN) to $1.8 \mathrm{~mm}$ diameter glass coverslips (Potomac Photonics, Halethorpe, MD). A $1.8 \mathrm{~mm}$ diameter craniotomy was made centered on the previous injection site with a taper pointed-drill (Henry Schein Inc, 9004367) and the dura was removed with a micro curette (FST, 10080-05). The overlying cortex was gently aspirated to reveal capsular fibers 
during continuous irrigation with ice-cold aCSF solution and bleeding was controlled with a collagen gel sponge (Avitene). Under minimal bleeding, a $30 \mathrm{~g}$ blunt syringe was used to gently aspirate capsular and CA1 alveus fibers with white appearance and CA1 pyramidale and moleculare with pink appearance until vasculature of the hippocampal fissure became visible (under bright light with low bleeding). The imaging implant, attached to the stereotactic handle, was then gently lowered into the craniotomy and affixed to the skull using dental cement (Unifast Trad powder and LC light cured acrylic UV, Henry Schein).

\section{Behavioral training and apparatus} $(1 \mathrm{ml}$ per day) and trained to run on treadmill while head-restrained. The training period typically lasted 7-10 days ( 2 training sessions/day, $15 \mathrm{~min}$ each) until the mice were able to run for at least $1 \mathrm{lap} /$ minute and seek reward from 3 randomly placed reward zones by licking the water delivery port. We then initiated the motorized belt adjusted to the natural velocity of each mouse and proceeded training for 2-3 more days. We did not utilize mice that were not getting all of their daily water supply during treadmill training, and were not motivated to move on the treadmill. A subset of mice preformed treadmill running without the help of motorized belt (Supplementary Figure 1a).

The behavioral apparatus consisted of a $2 \mathrm{~m}$ long, 3" wide fabric belt stretched between 6" diam. laser-cut plastic wheels, mounted on an aluminum frame (8020.net). Spatial triggering of task events was performed by custom software via serial communication with a microcontroller (Arduino DUE) and an associated circuit board (OpenMaze OM4 PCB, www.openmaze.org) on the treadmill. The axle of the treadmill wheel was attached to a quadrature rotary encoder (US Digital part \#: MA3-A10-125-B) connected to a custom quadrature decoder board and Arduino Nano (courtesy of Wen Li). Angular displacement was converted into a linear distance based on the circumference of the treadmill. The errors were corrected via a registration anchor marked by radio-frequency identification (RFID) buttons (SparkFun Electronics) at the lap boundary of the belt and was detected when it passed over an RFID reader (ID-12LA, SparkFun) affixed underneath the mouse. A $12 \mathrm{~V} \mathrm{DC}$ gear motor was attached to the axle of the treadmill connected to a separate Arduino/OpenMaze shield using pulse-width modulation to adjust the rotation speed. A water reservoir connected to a water delivery port consisting of a small gavage needle (Cadence Science) was placed within reach of the mouse's tongue. A capacitance touch sensor (Sparkfun \#MPR121) was attached to the water port to measure licking and the sensor was connected to the Arduino/OM4 PCB. Small 5-10 $\mu$ l drops of water were delivered by the brief opening a solenoid valve (Parker Hannefin) connected to the water port and controlled by the OM4 H-bridge circuit. Rewards were triggered at random locations each lap when mice entered a $10 \mathrm{~cm}$ long reward zone on the track and were available until mice exited the reward zone or 3 sec had elapsed. Olfactory stimuli consisted of undiluted isoamyl acetate (IAA, Sigma W205532) which was added to syringe filters (Whatman \#6888-2527) and delivered by opening a solenoid valve (SMC) connected to a flow controller delivering constant airflow of compressed medical grade air for $1 \mathrm{~s}(\sim 3 \mathrm{psi})$, controlled by another OM4 H-bridge output. Visual and tactile stimulation consisted of a red LED contralateral to the imaged region, or a 1" square piece of sand paper brushed by the contralateral whiskers using a stepper motor, at approximately the speed of the treadmill belt. A custom-written B-Mate behavioral software application implemented in Java was 
used for recording mice's licking, its position on the belt, and cue delivery. Mice were monitored using an IR camera (PS3eye) and illuminated using an IR LED array.

To isolate cue-selective responses among the granule cell population, normal cue laps in which the olfactory, visual, or tactile cue was presented in the middle of the treadmill track $(90-110 \mathrm{~cm})$ were interspersed with occasional laps $(10-20 \%$ of laps) in which the same cue was omitted ("omit" laps), or shifted forward $1 / 4$ of the track ( $0.5 \mathrm{~m}$, "shift" laps). For a subset of sessions, the olfactory cue was presented at one of 5 locations along the track randomly each lap, in order to examine the effect of spatial pairing of the cue. In all experiments, the treadmill belt material was changed between sessions to reduce the chances of urine contamination which might act as an additional olfactory cue.

\section{In vivo two-photon imaging} Imaging was conducted using a microscope setup which consists of $8 \mathrm{kHz}$ resonant galvanometer scanner (Bruker) mounted to a mirror-based multi-photon microscopy system (Prairie Technologies) and an ultra-fast pulsed laser beam (920-nm wavelength; Chameleon Ultra II, Coherent, 20-40-mW average power at the back focal plane of the objective) controlled with an electro-optical modulator (Conoptics, Model 302 RM). GCaMP fluorescence was excited through a 40x water immersion objective (Nikon NIR Apo, $0.8 \mathrm{NA}, 3.5 \mathrm{~mm}$ WD) and fluorescence signals detected with photomultiplier tubes (Hamamatsu 7422P-40), acquired with PrairieView software (Prairie) at $30 \mathrm{fps}$ frame rate $(512 \times 512$ pixels, $1.3 \mathrm{~mm} / \mathrm{pixel})$. A custom dual stage preamp $\left(1.4 \times 10^{5} \mathrm{~dB}\right.$, Bruker) was used to amplify signals prior to digitization. Two goniometers (Edmund Optics) were used to adjust the angle of each mouse's head in order to achieve the same imaging plane over multiple sessions.

Data processing for $\mathrm{Ca}^{2+}$ imaging. Movies were motion corrected using the NoRMCorre algorithm using a non-rigid registration method that splits the field of view (FOV) into overlapping patched that are registered separately then merged by smooth interpolation ${ }^{22}$. Videos were then spatially and temporally down-sampled by 2 to reduce noise and reduce the computational power required for cell segmentation. Spatial and temporal components for individual cells were extracted using large-scale sparse non-negative matrix factorization ${ }^{23}$ or using the singular value decomposition method by the Suite2p algorithm (https://github.com/cortex-lab/Suite2P), both of which resulted in similar regions of interest (ROIs). We used Suite2p graphical user interface to manually select small, densely packed DG granule cells and discard large isolated cell bodies corresponding to mossy cells or other hilar interneurons. To obtain the total number of DG granule cells within the imaging fields of view in a subset of total sessions, time averaged images were segmented using the Cellpose algorithm (https://github.com/MouseLand/cellpose) followed by manual inspection. $\mathrm{Ca}^{2+}$ transient events were defined by a custom detection algorithm which identifies fluorescence peaks with a rise slope greater than 4 standard deviations above an iteratively refined baseline.

Behavioral and Imaging Data Alignment. Behavioral data were aligned to $\mathrm{Ca}^{2+}$ data using the record of a synchronization signal between the two computers used for data collection. Behavioral data were resampled to match $\mathrm{Ca}^{2+}$ imaging data frame times.

716 
718 Data were analyzed using custom-written routines implemented in MATLAB. Plots were generated in MATLAB and Prism and edited in Adobe Illustrator CC 2018.

Identification of spatially-tuned neurons. We restricted our analysis to data from epochs of continuous running at least $2 \mathrm{sec}$ in duration and with a minimum peak speed of $5 \mathrm{~cm} / \mathrm{sec}$. For each lap, position data and $\mathrm{Ca}^{2+}$ transient events for each cell were binned into $2 \mathrm{~cm}$-wide windows (100 bins), generating raw vectors for occupancy-by-position and calcium transient numbers-by-position which were then circularly smoothed with a Gaussian kernel $(S D=5 \mathrm{~cm})$. A firing rate-by-position vector was computed by dividing the smoothed transient number vector by the smoothed occupancy vector. Within each lap, we circularly shuffled the positions 1000 times and recomputed firing rate-by-position vectors to generate a null distribution for each spatial bin. A spatially selective cell was defined that met the following criteria: (a) the cell should fire above its mean firing rate within its spatial field in at least $20 \%$ of laps or for a minimum of 3 laps; and (b) observed firing should be above $99 \%$ of the shuffled distribution for at least 5 consecutive spatial bins $(10 \mathrm{~cm})$ wrapping around the two edges of the belt. We identified spatially tuned neurons by excluding laps in which sensory cues were omitted or shifted and calculated firing rate vectors in these laps separately. Cells were classified as cue or place cells based upon their peak firing location and activity during laps in which the cue was omitted. Among all of the spatially tuned neurons, "middle cue cells" were defined as those with averaged spatial fields that overlapped with at least $50 \%$ of the $45^{\text {th }}$ and $55^{\text {th }}$ bins and had peak amplitude at least two times larger than those in cue-omitted laps. "Lap-cue cells" were defined as those with averaged spatial fields overlapping at least $50 \%$ of the region wrapping around the $90^{\text {th }}$ and $10^{\text {th }}$ bins in the normal laps. The remaining cells constituted the "place cells".

Spatial information, stability, consistency, and emergence of spatial fields. To calculate a measure for spatial information content for granule cells in Figure 1, we adapted a traditional method of spatial information assessment ${ }^{21,51}$ to $\mathrm{Ca}^{2+}$ imaging data. For each cell, we used the firing rateby-position vector and shuffled null distribution, as computed above, and calculated the spatial information content for each as described previously ${ }^{51}$. To account for the fact that low firing rates artificially produce high spatial information scores, we subtracted the mean of the shuffled information per spike from observed information per spike, divided by the standard deviation of the shuffled values to determine the spatial variance for each cell. Therefore, the amount of spatial information is inferred from differences in normalized $\mathrm{Ca}^{2+}$ activity in each neuron and reported as bits per s. To measure spatial field correlation across environments, after identifying spatially tuned neurons, we calculated a Pearson's correlation coefficient between a cell's averaged firing rate-by-position vector in different sessions. The consistency of place field firing was determined as the cross-correlation between the averaged firing rate-by-position vector of normal cue laps from the first and the second halves of the session. We determined place field onset lap in normal cue laps (Figure 1) as described previously ${ }^{34}$. Briefly, starting on lap 1 we searched for a significant $\mathrm{Ca}^{2+}$ transient event present within the boundaries of the previously determined mean spatial field calculated from all the laps in the session. If one were found we would then search for $\mathrm{Ca}^{2+}$ transient event on each of the next 4 laps. If 3 of the 5 laps had $\mathrm{Ca}^{2+}$ transients within the mean place field boundaries, lap 1 would be considered the place field onset lap. If either lap 1 
had no $\mathrm{Ca}^{2+}$ transient or less than 3 of the 5 laps had $\mathrm{Ca}^{2+}$ transient, we would move to lap 2 and repeat the search. To determine place field onset lap in cue shifted laps (Figure 1), we searched for the first $\mathrm{Ca}^{2+}$ transient event on 2 of 4 consecutive cue shifted laps.

Multi-Session Cell Tracking. Cells were tracked across sessions using CellReg ${ }^{26}$. Briefly, rigid alignment with both translations and rotations was performed on spatial footprint projections of each session and manually inspected for quality. To improve performance with our data, we modified the CellReg source code to consider complete spatial footprints instead of centroids during alignment. The centroid distance between neighbors was then calculated and used to create a probabilistic model that estimated the expected error rate at different thresholds. The optimal centroid distance threshold was chosen by the algorithm and used to match cells. A clustering algorithm then refined these decisions previously made using pairwise comparisons.

Following cell registration, tracked cells were matched with their corresponding functional cell types (i.e. mid-, lap-cue, place cells, as described above). All analyses presented in Figure 2 are carried out in pairwise, to maximize the number of cells in each comparison and to minimize the total number of comparisons. For multiday comparisons we used Day1, session 1 as the normal session, and for multisession comparisons we used the visual stimulus session as the reference. To calculate the fraction of cells that maintain their identity, cell pairs that were counted as being the same cell type in both sessions was divided by all of that cell type in the normal session. In order to derive a null distribution for preservation of pairwise identity, we randomly permutated the cell IDs of all the tracked cells in pairwise sessions 1000 times and calculated the fraction of cells that were the same, among all of that cell type in the normal session. We calculated $p$-values by comparing actual data to this null distribution, $97.5^{\text {tho }} \%$ of the null distribution is presented dotted lines in Figure 2.

Spatial event rate and Population vector (PV) correlation analysis. Comparison of the activity between different sessions was calculated using Pearson's correlation of the spatially binned, averaged firing rate-by-position vector in cue normal laps of all recorded cells in 8 mice for multiday analysis ( 1 session from each condition) and 6 mice for multisession analysis ( 1 session from each condition) in Figure 2 . The variability in neural activity between lap types was calculated by using Pearson's correlation on each $2 \mathrm{~cm}$ bins of the firing rate-by-position vector along the treadmill during odor cue trials (mean for all spatially tuned cells, 4,091 total spatially tuned cells from 8 mice across 66 sessions).

\section{Spatial modulation}

To compare single cell cue responses at different locations, cue-triggered average z-scored calcium transients were measured for cue cells, defined based upon omit laps as done previously. For dual cue location experiments, cue cells were identified as cells that whose cue-zone calcium event rate was greater than $95 \%$ of shuffled responses versus the cue-omitted laps. Cue cells that significantly preferred one location were counted when responses at one cue location exceeded $95 \%$ of the shuffled cue position event rates. Spatial modulation index in this paradigm is listed as a ratio of mean spatial firing rates for the nonpreferred location over the preferred 
Bayesian Reconstruction Analysis. To calculate the probability of the animal's position given a short time window of neural activity, we used a previously published method based on Bayesian reconstruction algorithm ${ }^{25}$. Briefly, $\mathrm{Ca}^{2+}$ transient events for each cell were binned into 1 second windows to construct firing rate vectors. For each of these binned firing rate vectors, Bayesian classification of virtual position (posterior probability for each bin) was performed by a previously described method ${ }^{25}$ utilizing a template comprising of a cell's smoothed firing rate-by-position vectors. In order to cross-validate our decoding procedure, we divided firing rate-by-position template into lap crossings, used $1 / 5^{\text {th }}$ of laps as "testing" dataset while the remaining $4 / 5^{\text {th }}$ of laps constituted the "training" dataset. For example, lap 1 was tested based on the firing rate-byposition vectors calculated using laps $2,3,4,5$, lap as template, and lap 6 was tested based on the firing rate-by-position vectors calculated from laps $7,8,9,10$, and so on. The resulting posterior probability distribution for each bin is the likelihood for an animal is located in that bin, which adds up to 1 , and the bin with the maximum posterior probability is the estimated position of the animal. To determine the decoding error we calculated the absolute difference between the animal's actual position and the maximum posterior probability in that bin. Post-reconstruction, we divided the time bins (excluding those with no activity) according to the lap types.

\section{Cue shift analysis}

Spatial firing rates for each spatially tuned cell on normal middle cue laps were cross-correlated with firing rates on shift laps in order to estimate the shift magnitude for each cell after cue manipulation. Binned histograms of numbers of cells with peak firing rates at particular locations along the track were plotted with respect to their shift magnitudes on cue shift laps. The spatial distribution of cells showing no shift ( $<5 \mathrm{~cm}$ shift, "non cue-influenced") or that shifted their firing along with the cue $(-50 \mathrm{~cm}+/-5 \mathrm{~cm}$, "cue influenced") was then compared.

\section{Inhibition analysis}

Out-of-field firing was calculated for spatially tuned cells as the spatial event rate, excluding the peak place field for that cell $(+/-10 \mathrm{~cm})$. Average out-of-field firing rates were then calculated across all cells for normal middle cue laps and intermittent laps where the cue was omitted. For comparison of cue-associated excitation and inhibition levels, average firing rates were computed by session for the $20 \mathrm{~cm}$ region surrounding the middle cue, with respect to the normal pre-cue baseline firing rate. Cue-related inhibition of place cell firing was calculated by selecting spatially tuned cells whose firing field on normal middle cue laps fell within the region of the cue on shift laps $(50-80 \mathrm{~cm})$. Firing rates for these cells were then averaged for normal laps where the cue was not presented in this location and compared with laps where the cue was shifted to this region $(50 \mathrm{~cm})$. Mutual inhibition between cues was calculated by first selecting cue cells responsive to each of 2 cues of different modalities (olfactory or visual, presented at $40 \mathrm{~cm}$ and $120 \mathrm{~cm}$, respectively) based upon their activity when each cue was omitted, as described above for single cues. Responses were then averaged for each cue cell for laps in which the cues were presented alone at these locations versus intermittent laps where the cues were presented together at one of the two locations.

\section{Data and Software Availability}

Data and custom programs are available upon reasonable request. 


\section{Figures}
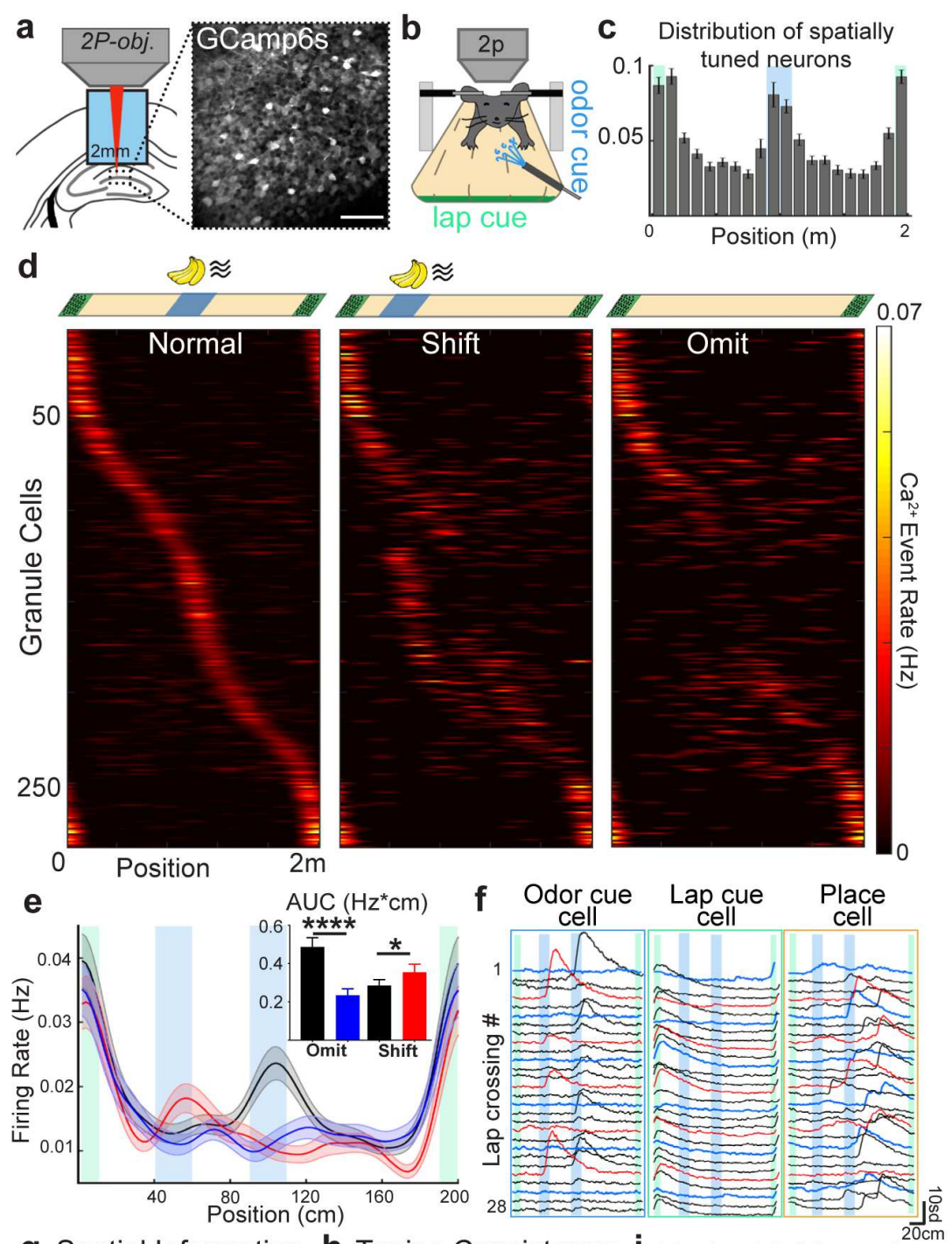

g Spatial Information $\mathbf{h}$ Tuning Consistency $\mathbf{i}$ Tuning Field onset
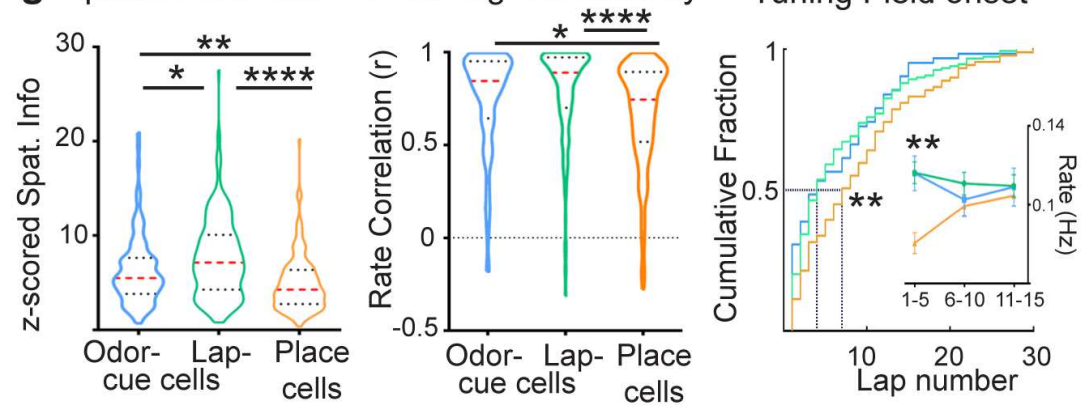

Figure1: Robust representation of sensory cues in the dentate gyrus

a) Two-photon imaging of DG population calcium activity. Left, diagram of the imaging window implant in the dorsal dentate gyrus. Right, Time averaged in vivo two-photon image of GCaMP6sexpressing granule cells.

b) Diagram of head-fixed treadmill apparatus for spatial sensory cue delivery. 
c) Fraction of spatially tuned cells at each linearized treadmill position (bins $=10 \mathrm{~cm}$ ) per session, $n=4091$ cells from 8 mice across $6-9$ sessions/mouse, locations of the odor and lap cue are shown blue and green shaded areas, respectively.

d) Spatial patterns of DG neuron activity during cue task. Top: Location of lap cue (green boxes) and an odor cue (blue box), on normal, shift or omit laps. Bottom: Lap-averaged spatial firing rates of 285 spatially tuned neurons ( $n=8$ mice) during their first session with the cue on normal middle location (left), cue-shifted (middle) and cue-omitted (right) laps. Each row across all graphs represents a single cell, and the $x$ axis represents the treadmill position.

e) Average firing rates by position for neurons shown in panel D on normal (black), cue-shifted (red) and cue-omitted laps (blue, mean \pm SEM). (Inset) averaged area under the firing rate curves $\left(\mathrm{Hz}{ }^{*} \mathrm{~cm}\right)$ within the middle cue region during normal (black bar), cue omitted (blue bar), and cue shifted laps (red bar). $P_{\text {normal-omit }}<0.0001, P_{\text {normal-shift }}=0.02$, Wilcoxon matched-pairs signed rank test, error bars are mean \pm SEM.

f) Example fluorescence traces from odor-cue, lap-cue and place cells within a single session. Black, red, and blue traces represent normal, cue shifted and omitted laps, respectively. Scale bars are $\mathrm{cm}$ and standard deviation from each cell's baseline fluorescence.

g) Z scored spatial information of cue and place cell populations. Z scored spatial information, $\mathrm{X}^{2}=48.47, \mathrm{p}<0.0001$, PodorCue-LapCue $=0.0387$, PodorCue-Place $=0.0028, P_{\text {LapCue-Place }}<0.0001$.

h) Tuning consistency of cue and place cells. Firing rate correlation between first and last halves of the session, $X^{2}=32.60, p<0.0001, P_{\text {OdorCue-LapCue }}=0.2224, P_{\text {OdorCue-Place }}=0.0117, P_{\text {LapCue- }}$ Place $<0.0001$.

i) Emergence of cue and place responses. Cumulative distribution of spatial field onset lap in normal laps for cue and place cells, $x^{2}=9.29, p=0.0096$, PodorCue-LapCue $=0.8925$, POdorCuePlace $=0.0194$, $P_{\text {LapCue-Place }}=0.0091$. (Inset) Spatial field firing rates of cue and place cells during $1-5^{\text {th }}, 6-10^{\text {th }}$ and $11-15^{\text {th }}$ laps, main effect of cell type: $F_{2,1473}=6.73, p=0.0012$; cell type $\times$ lap number interaction $F_{4,1473}=2.44, p=0.04$; main effect of lap number: $F_{2,1473}=0.28, p=0.7$; Lap 1-5 ${ }^{\text {th }}$, POdorCue-LapCue $=0.99$, POdorCue-Place $=0.0011, P_{\text {LapCue-Place }}<0.0001,2$-way ANOVA and Tukey's multiple comparisons test.

NodorCue $=114, N_{\text {LapCue }}=220, N_{\text {PlaceCell}}=160$, from 8 mice 2 sessions each. Red dotted lines in violin plots show median, black dotted lines show quartiles. Comparisons are Kruskal Wallis and Dunn's multiple comparisons tests unless otherwise noted. ${ }^{*} P<0.05 ;{ }^{* *} P<0.01$; ${ }^{* *} P<0.001$.

See also Supplementary Figures 1-4. 
a Stability over time

b Stimulus selectivity
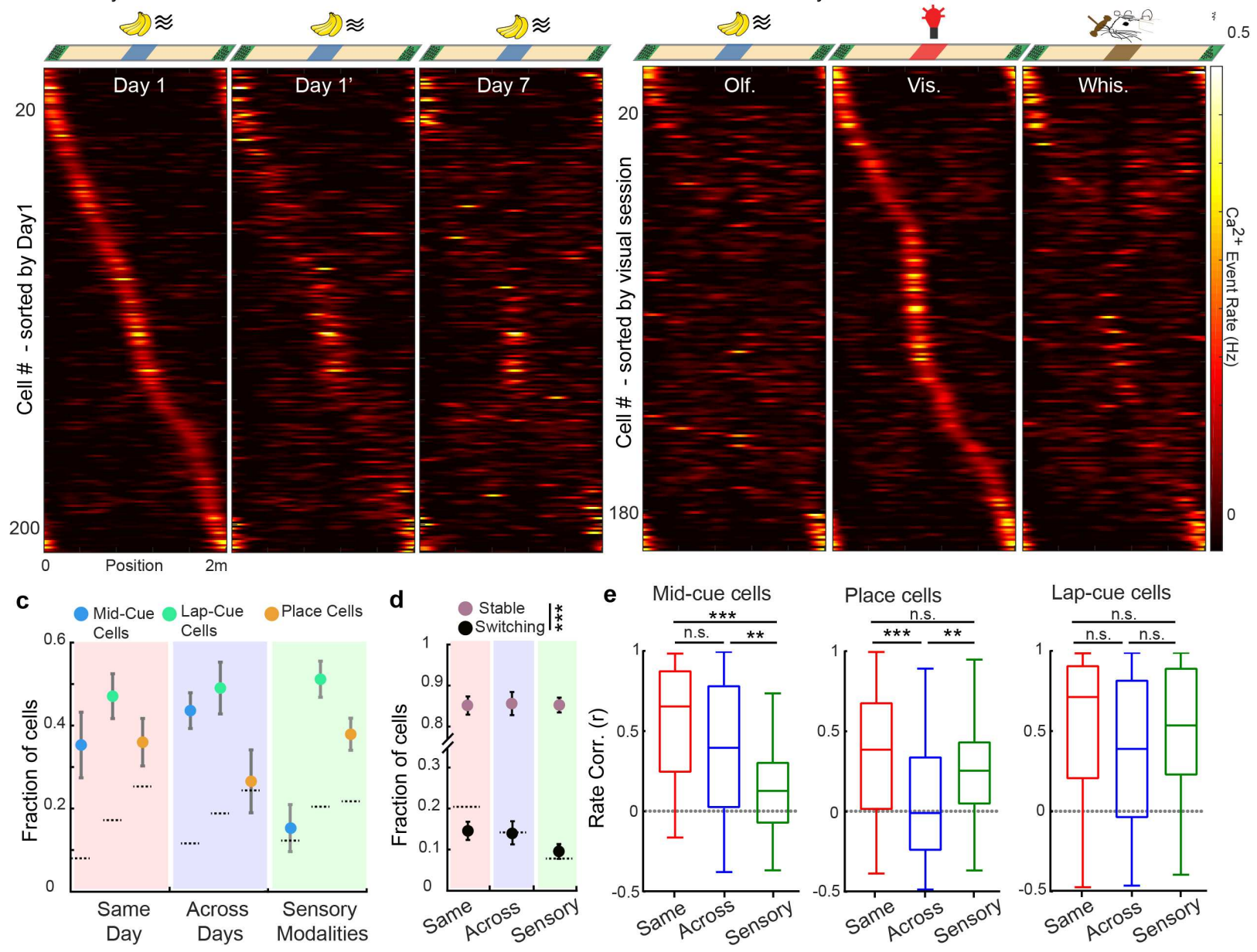

e Mid-cue cells

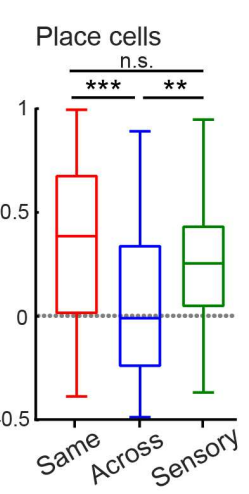

Lap-cue cells

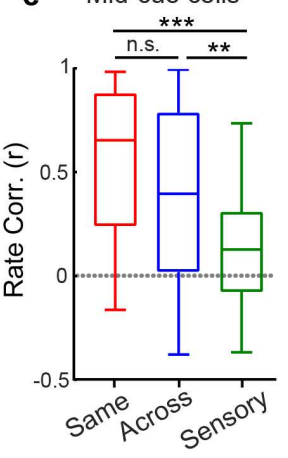

g
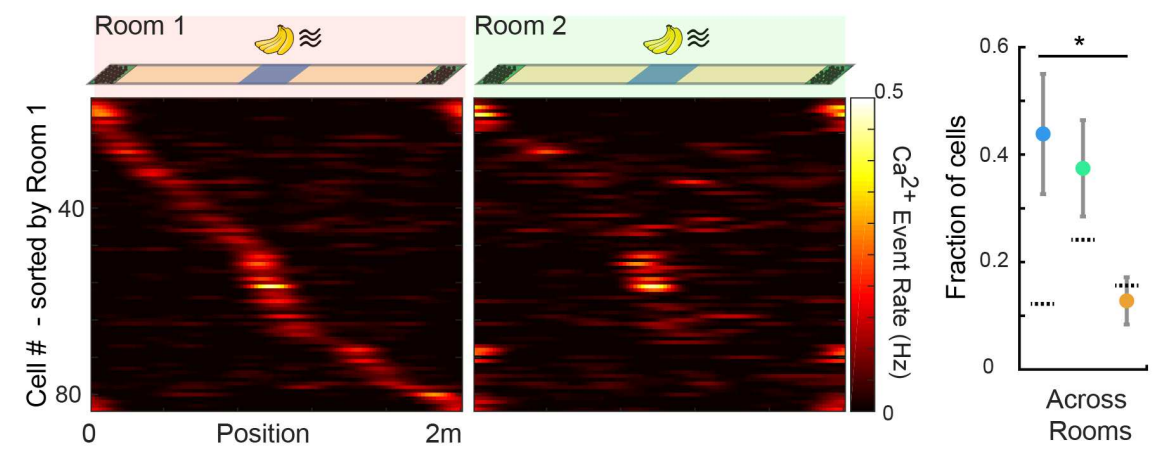

h

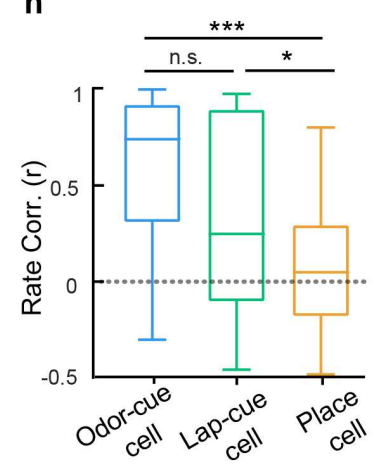

Figure 2: Sensory cue and place responses differ in stability and selectivity

a) Spatial firing rates of individual DG granule cells matched between sessions within a day and over one week. Top: diagram of odor cue presentation during all sessions. Bottom: Spatial firing rates for spatially tuned neurons tracked during subsequent sessions on the same day or one week later, ordered according to the position of peak activity during the first exposure $(n=233$ cells, 8 mice).

b) Activity of granule cells in response to different sensory cues. Top: diagram of behavior in sessions with cues of different sensory modalities in the middle position and an invariant lap cue. 
Bottom: Spatial tuning for neurons tracked through consecutive sessions during exposures to different cues. Data are shown for neurons with significant tuning in visual cue session and tracked in olfactory and whisker tactile cue sessions ( $n=196$ cells, 6 mice).

c) Fraction of cross-session registered cells that encoded the same variable, lap cue (green), middle cue (blue), or place (orange), within same day (left), across days (middle), and during exposures to different sensory modalities (right). Cells encoding the same variable on the same day, $X^{2}=1.81, p=0.4039, P_{\text {LapCue-OdorCue }}=0.4221, P_{\text {LapCue-Place }}=0.549$, PodorCue-Place $=0.9713$; cells encoding the same variable across days, $X^{2}=14.36, p<0.001, P_{\text {LapCue-OdorCue }}=0.1566, P_{\text {LapCue- }}$ Place $<0.0001$, PodorCue-Place=0.0313 (8 mice, 8 matched sessions, Day1-Day1', Day1-Day7); cells encoding the same variable with different sensory modalities, $X^{2}=19.78, p<0.00001, P_{\text {LapCue- }}$ OdorCue $<0.00001$, $P_{\text {LapCue-Place }}=0.0766$, POdorCue-Place $=0.0464$ (6 mice, 12 matched sessions, Odor-Vis. and Vis.-Tact.); Kruskal-Wallis test, Dunn's multiple comparisons test. Dashed lines represent $97.5^{\text {th }} \%$ of null distributions for each cell type. Error bars, mean \pm SEM.

d) Fractions of spatially tuned cells that stably encode only one variable (cue or place, pink) or switched response types (cue to place or vice versa, black) within same day, across days and in response to different sensory cues: $P_{\text {Switch-Stable }}$ (Same Day) $<0.001, P_{\text {Switch-Stable }}$ (Across Days) $<0.001$, $P_{\text {Switch-Stable (Sensory Modalities) }}<0.001$, Wilcoxon Rank Sum test. Dashed lines: $2.5^{\text {th }}$ and $97.5^{\text {tho }} \%$ of null distributions. Error bars, mean \pm SEM.

e) Mean rate correlations within the same day (red), different days (blue) and different sensory cues (green) for mid-cue cells (left, $X^{2}=27.88, p<0.0001, P_{\text {Same Day-Across Days }}=0.2465, P_{\text {Same Day- }}$ Sensory Modalities $<0.0001, P_{\text {Across Days-Sensory Modalities }}=0.0038, N_{\text {Same Day }}=37, N_{\text {Across Days }}=37, N_{\text {Sensory }}$

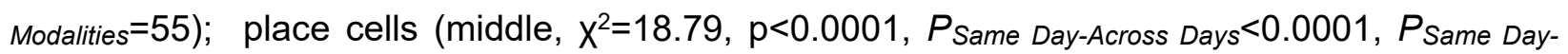
Sensory Modalities $=0.7449, P_{\text {Across Days-Sensory Modalities }}=0.0055, N_{\text {Same Day }}=76, N_{\text {Across Days }}=76, N_{\text {Sensory }}$ Modalities $=83$ ), lap-cue cells (right, $X^{2}=5.096, p=0.0782$, PSame Day-Across Days $=0.0723$, P Same DaySensory Modalities $>0.9999, P_{\text {Across Days-Sensory Modalities }}=0.7932, N_{\text {Same Day }}=80, N_{\text {Across Days }}=80, N_{\text {Sensory }}$ Modalities $=59$ ). Kruskal-Wallis test, Dunn's multiple comparisons test. Boxes, 25th to 75th percentiles; bars, median; whiskers, 99\% range. ${ }^{*} P<0.05$; ${ }^{* *} P<0.01$; ${ }^{* * *} P<0.001$.

f) Activity of DG granule cells in response to the same odor-cued track when recorded in different rooms. Top: diagram of exposures to an odor in the middle position and an invariant lap cue, performed in different rooms. Bottom: Spatial firing rates for tuned neurons tracked through consecutive sessions in different rooms ( $n=83$ cells, 4 mice).

g) Fraction of cross-session registered cells that encoded the same variable, odor cue (blue), lap cue (green) or place (orange) in different recording rooms. $x^{2}=6.59, p=0.0372$, PodorCuePlace $=0.0490, P_{\text {LapCue-OdorCue }}=0.9557, P_{\text {LapCue-Place }}=0.0972$. Dashed lines: $2.5^{\text {th }}$ and $97.5^{\text {th }} \%$ of null distributions. Error bars, mean \pm SEM.

h) Comparison of spatial firing rate correlations in all tuned cells across rooms $\left(X^{2}=15.85\right.$, $\mathrm{p}<0.001, P_{\text {Odor-Lap cue }}=0.27, P_{\text {Odor Cue-Place }}<0.001, P_{\text {Lap Cue-Place }}=0.03, N_{\text {Odor-Cue }}=25, N_{\text {Lap- }}$ $\left.\mathrm{Cue}=33, N_{\text {Place }}=26\right)$. Kruskal-Wallis test, Dunn's multiple comparisons test. Boxes, 25th to 75th percentiles; bars, median; whiskers, 99\% range. ${ }^{*} P<0.05$; ${ }^{* *} P<0.01$; ${ }^{* * *} P<0.001$. See also Supplementary Fig. 5. 


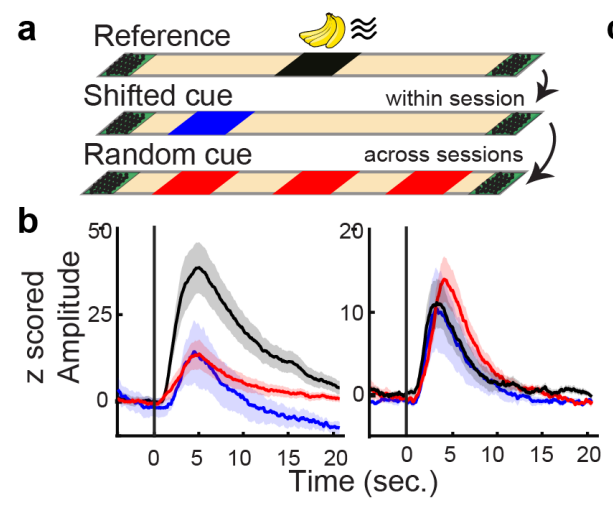

e

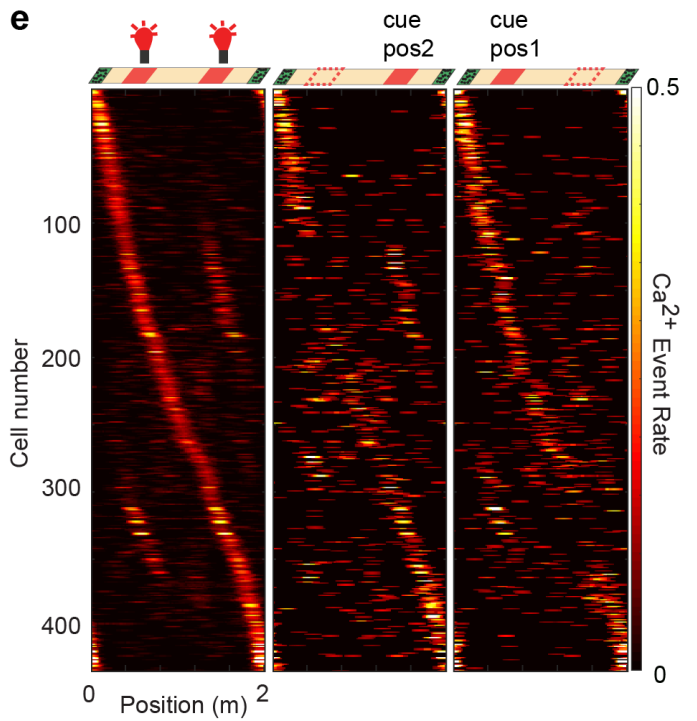

c Peak amplitude of cue reponses

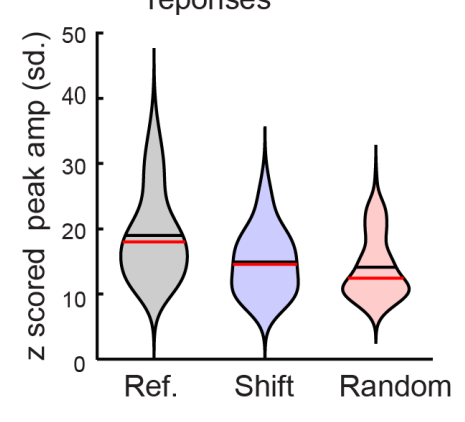

f Cue pos. 1 pref. cells

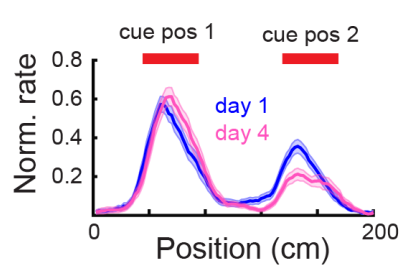

Cue pos. 2 pref. cells

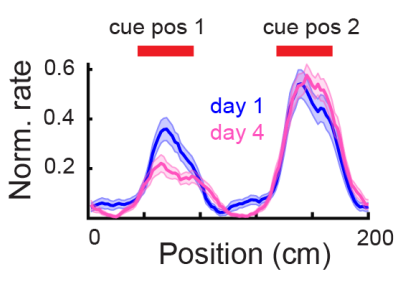

d Spatial modulation of cue reponses
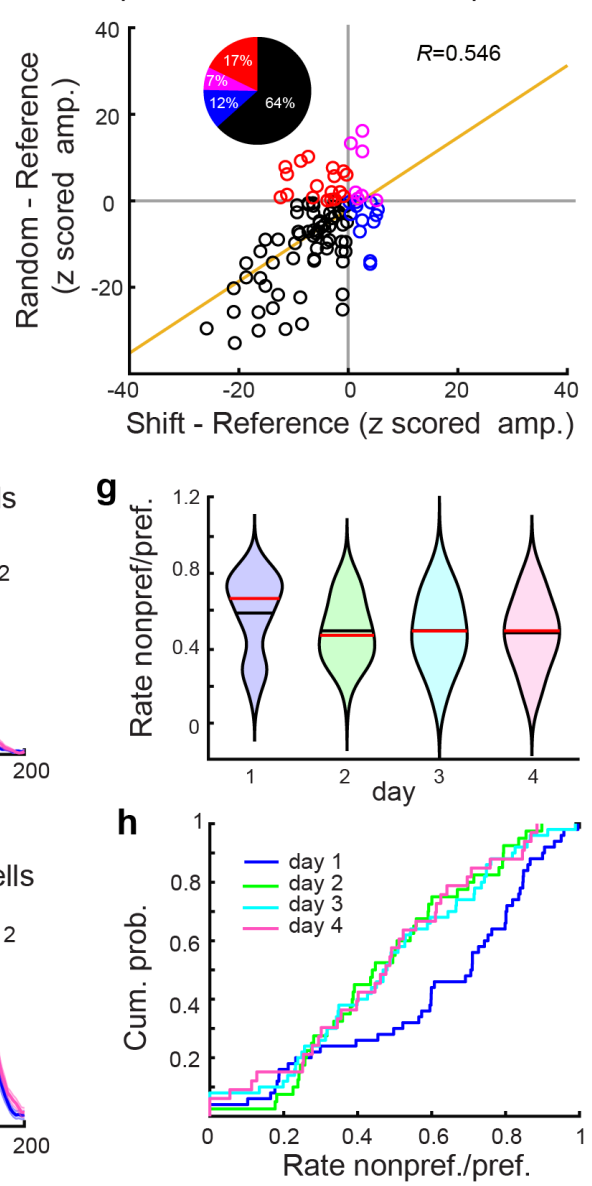

Figure 3: Sensory cue responses are spatially modulated

a) Experimental setup to examine effects of cue-location pairing on cue responses. Cue responses are measured with respect to a typical middle cue location or an intermittently shifted cue location in the same sessions, or with random administration of the same cue on each lap in a subsequent session.

b) Example cue-triggered average $\mathrm{Ca}^{2+}$ transients from two cue cells on normal (black), cueshifted (blue) and random-cue (pink) laps. Scale bars are sec and standard deviation from each cell's baseline fluorescence for the session.

c) Z-scored peak amplitudes of $\mathrm{Ca}^{2+}$ transients from odor cue cells in normal, shift and random cue presentation conditions; $X^{2}=49.36, p<0.001, P_{\text {Normal-Shift }}<0.0001, P_{\text {Normal-Random }}<0.0001, P_{\text {Shift- }}$ Random $>0.9999$, Friedman test and Dunn's multiple comparisons tests ( $n=101$ cells, 5 mice). Red lines in violin plots show median, black lines show mean.

d) Difference in the magnitude of cue responses in normal laps from random-cue and cue-shift laps. Yellow line, linear regression $\left(R^{2}=0.546, p=3.5 \times 10^{-9}\right)$. Pie chart shows the percentage of neurons with higher average responses in normal (black), cue-shift (blue), random cue presentation conditions (red), and both shifted and random laps (purple). 
e) (Top) Dual cue location task: LED visual cue is given consistently at two positions on the track. Arrangement of cues for normal laps (left, $80 \%$ of laps), laps where first cue is omitted (middle, $10 \%$ ), and laps where the second cue is omitted (right, 10\%). (Bottom) Spatial firing rates of 433 significantly tuned cells on first day of task ( $\mathrm{N}=3$ mice).

f) Average spatial firing rates for significant cue cells (vs. cue omitted laps), for cells preferring cue position 1 (top) or cue position 2 (bottom) on first day of task (blue, n=25 pos1, n=25 pos2) and 4 th day of task (pink, $n=13$ pos $1, n=20$ pos 2 cells).

g) Spatial modulation index (rate non-preferred cue/preferred cue) for all cue cells on days 1-4. ( $p=0.0125$, day 1 vs. days 2,3 , or $4, n=50 / 40 / 50 / 33$, Wilcoxon rank sum test).

h) Empirical cumulative probability distribution for all cue cells on days 1-4.

See also Supplementary Figure 6. 

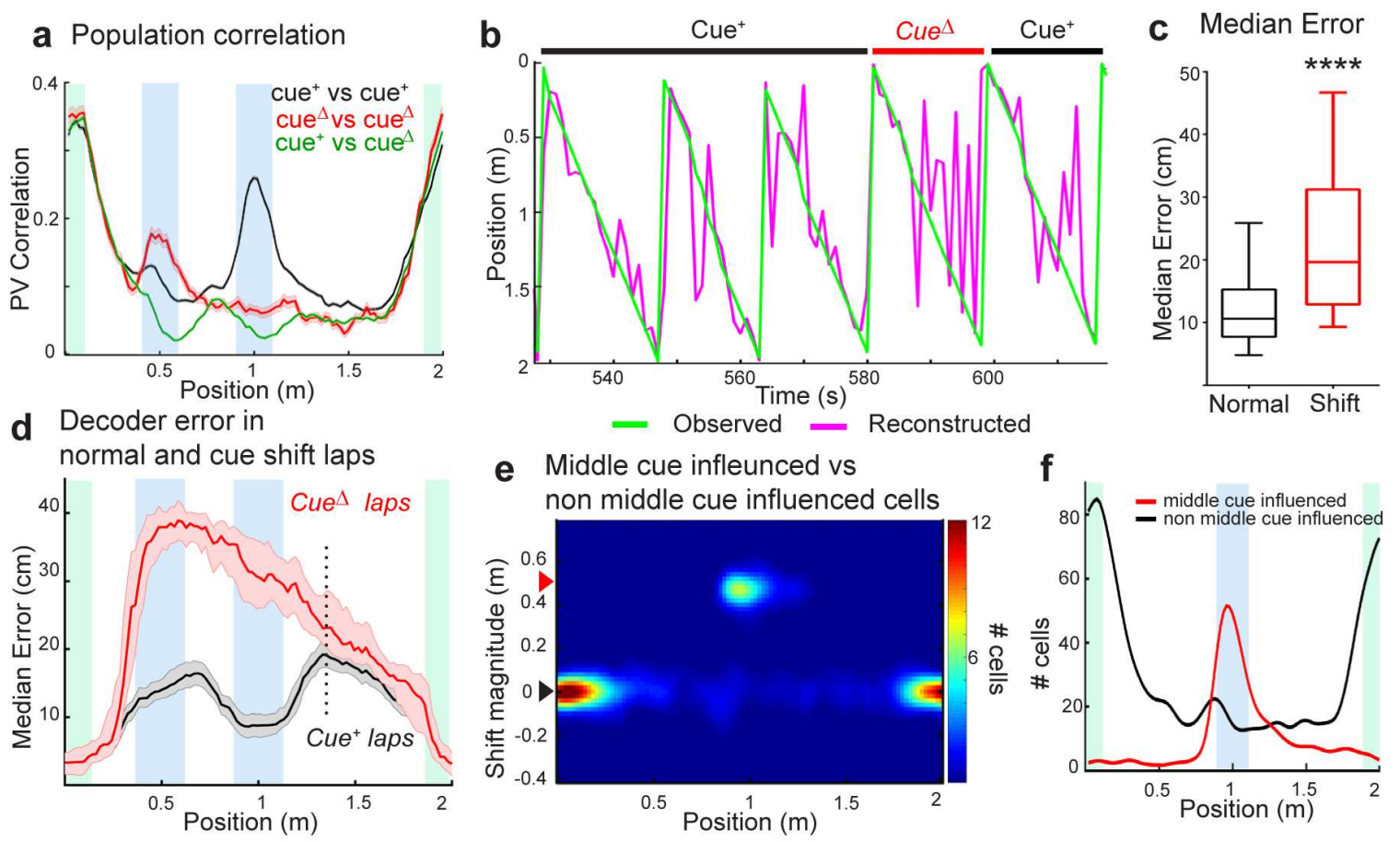

Figure 4: Cue shifts have limited effects on spatial encoding

a) Population vector correlations for all spatially tuned cells for normal middle cue laps and shifted cue laps in cue shift task. Locations of the middle/shift and lap cue are shown blue and green shaded areas, respectively.

b) Bayesian decoding of 4 normal and 1 cue shifted laps for a representative session, based upon activity from all spatially tuned granule cells. The magenta line shows predicted position at each bin, while the green line shows the observed position of the animal.

c) Average decoding error for normal and cue shifted laps calculated as the absolute median distance between the decoded value in each time bin and the actual value of the position $(p<0.0001$, Wilcoxon signed-rank test, $n=66$ sessions). Boxes, 25th to 75 th percentiles; bars, median; whiskers, $99 \%$ range.

d) Decoding error for each treadmill position in normal (black) and cue shifted laps (red). Shaded error region represents the difference of the median from the null distribution of median decoder error. Dotted line indicates point of statistical equality between normal and cue shifted laps.

e) Distribution of cell firing location shift on shifted cue laps for all spatially tuned cells, plotted based upon normal tuning location $(n=66)$. Red arrowhead indicates cue shift distance, and thus marks cells that are directly influenced by the cue. Black arrowhead indicates cells with zero shift, and thus not influenced by the cue.

f) Mean number of cells not influenced by the odor cue (shift magnitude $<0.05 \mathrm{~m}$ ) and cells shifting precisely along with the cue (shift magnitude $0.5 \mathrm{~m}+/-0.1 \mathrm{~m}$ ) at each track position (corresponding to cells in regions of black and red arrowheads in "b", respectively). Cue influenced cells are largely confined to locations around the cue itself, while non cue-influenced place cells are more evenly distributed, with a slight enrichment immediately preceding the middle cue.

See also Supplementary Figure 7. 

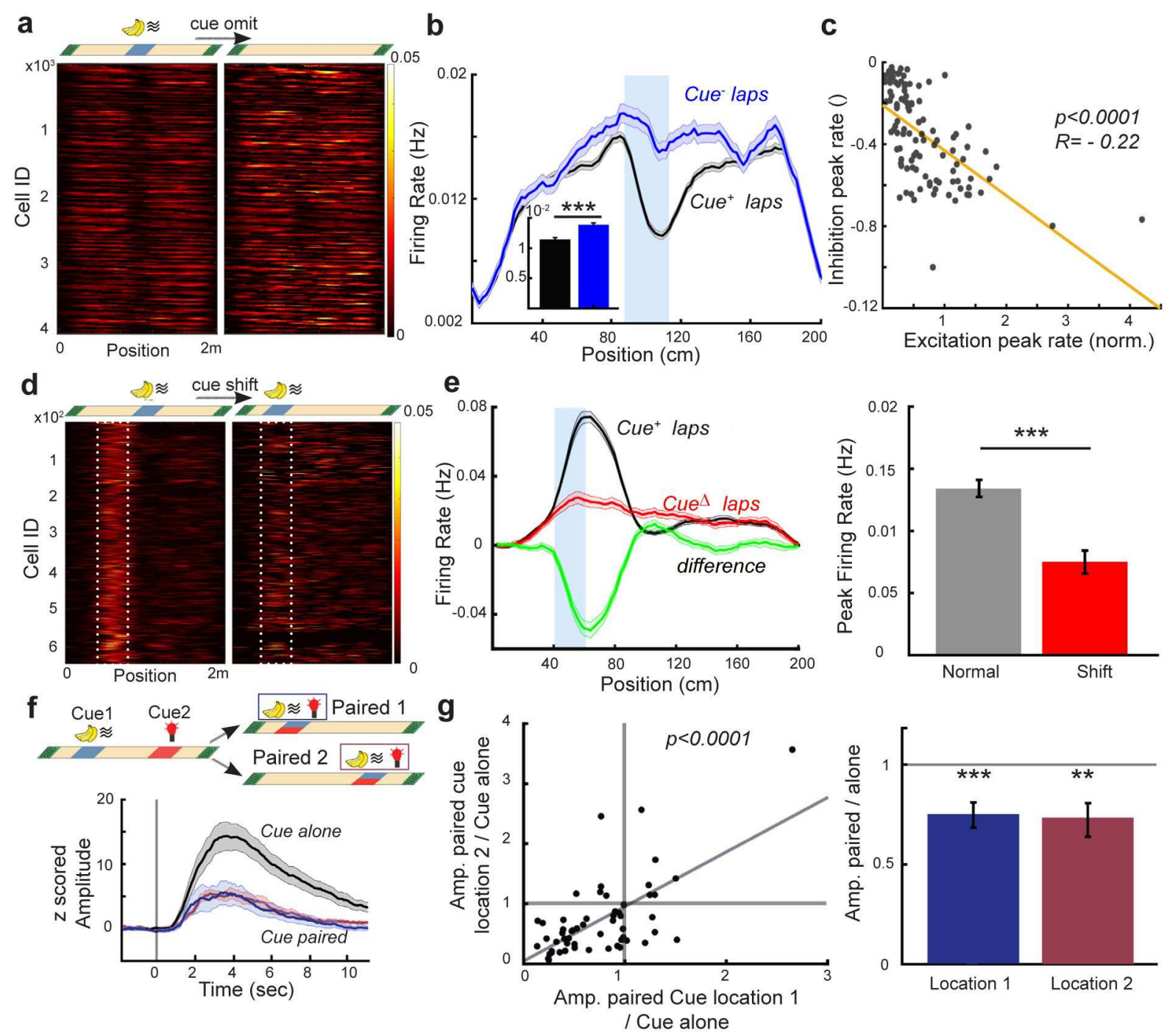

Figure 5: Cue presentation leads to suppression of diverse types of DG responses

a) Cue-related suppression of noisy, out-of-field firing. Spatial firing rates outside of the field center-of-mass $(+/-10 \mathrm{~cm})$ for significantly tuned cells during normal and cue omitted laps $(n=4,091$ cells from 66 sessions).

b) Average out-of-field firing rates from spatially tuned cells in the presence of the middle cue (black) compared to laps in which the cue is omitted (blue). Blue shaded area shows the cue delivery position. Inset, mean \pm SEM of firing rate, $p<0.001$, rank-sum test, $n=4,091$ cells.

c) Comparison of the magnitude of cue-related excitation and inhibition. Session averaged in-field firing rate vs. out-of-field firing rate ( 66 sessions). Yellow, linear regression: $p<0.0001, R^{2}=-0.22$.

d) Cue-related suppression of place cell firing. Spatial firing rates of place cells with firing fields at $40-80 \mathrm{~cm}$ during normal laps (left) and laps in which the cue is shifted to $40 \mathrm{~cm}$ (right).

e) (Left) Average firing rate by position for the above place cells in normal laps when the cue is not presented at this location (Cue ${ }^{+}$, black), compared with laps in which the cue is shifted to this location (Cue ${ }^{\Delta}$, red; diff. in green). (Right), Average peak firing rate of the same cells in "d" and "e", paired t-test, $p<0.001,645$ cells. 
f) Cue-related suppression of cells responding to other cues. (Top) diagram of intermittent cue pairing experiment. Cues of two different modalities were presented at different locations, interspersed with paired presentation of these two cues at one of these locations. (Bottom) example cue-triggered averaged $\mathrm{Ca}^{2+}$ transients for a cue cell strongly active when the cue is presented alone (black) but with reduced responses when paired with a different cue, regardless of location (blue, pair location \#1, purple, location \#2).

g) (Left) Amplitudes of paired cue responses at two pairing locations with respect to the response to the cue alone. Gray line represents the diagonal, $p<0.0001, R^{2}=0.91, n=56$ cells, 3 mice. (Right) Average relative response amplitude at the two pairing locations (Location 1: $0.75 \pm 0.061, \mathrm{p} 1=$ $1.45 \times 10^{-4}$; Location 2: $0.73 \pm 0.086, \mathrm{p} 2=0.003$, Wilcoxon Signed Rank Sum test, bar plots are mean \pm SEM).

See also Supplementary Figure 8. 


\section{Supplementary Figures}
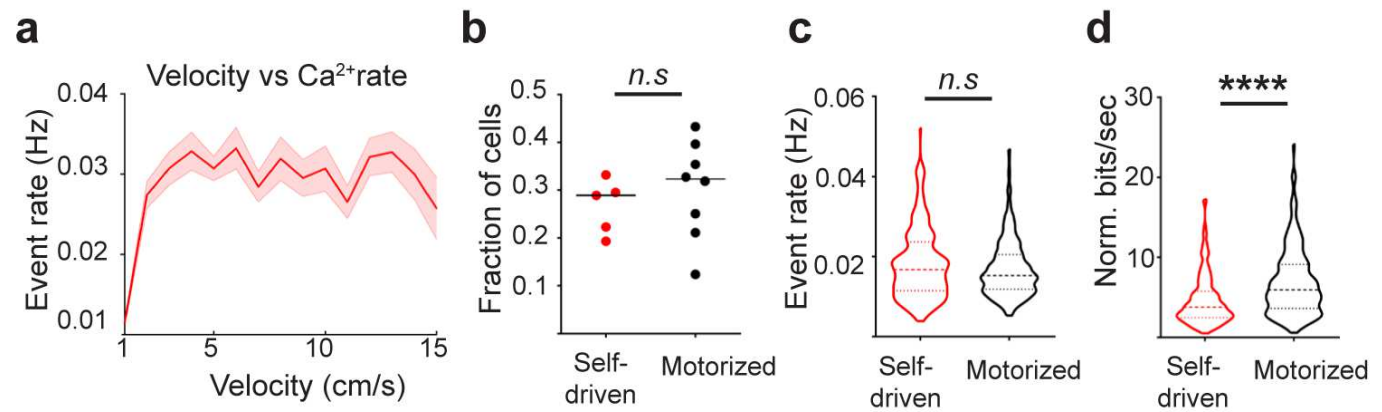

\section{Supplementary Figure 1. Motor driven treadmill does not influence spatial properties of DG neurons.}

a) DG population activity is dependent on the locomotion of the animal, shaded error bar represents mean $\mathrm{Ca}^{2+}$ events rate and SEM binned by normalized velocity (190 spatially tuned neurons in 5 mice, 1 session/mouse). b-d) Comparison of cell firing for self-driven vs. motorized treadmill. b) Fraction of active cells that are spatially tuned (with at least 0.001 transients per $s$ ) in mice advancing the treadmill belt through self-driven locomotion (black, 190 spatially tuned neurons in 5 mice, 1 session/mouse) and mice running on the motorized treadmill (red, 285 spatially tuned neurons in 8 mice, 1 session/mouse,) $p=0.435$, Wilcoxon Rank Sum test.

c) Firing rate of spatially tuned cells in self-driven vs. motorized treadmill $(p=0.1876$, Wilcoxon Rank Sum test).

d) Z scored spatial information in mice running on motorized treadmill vs. self-driven locomotion $(p<0.0001$, Wilcoxon Rank Sum test). 

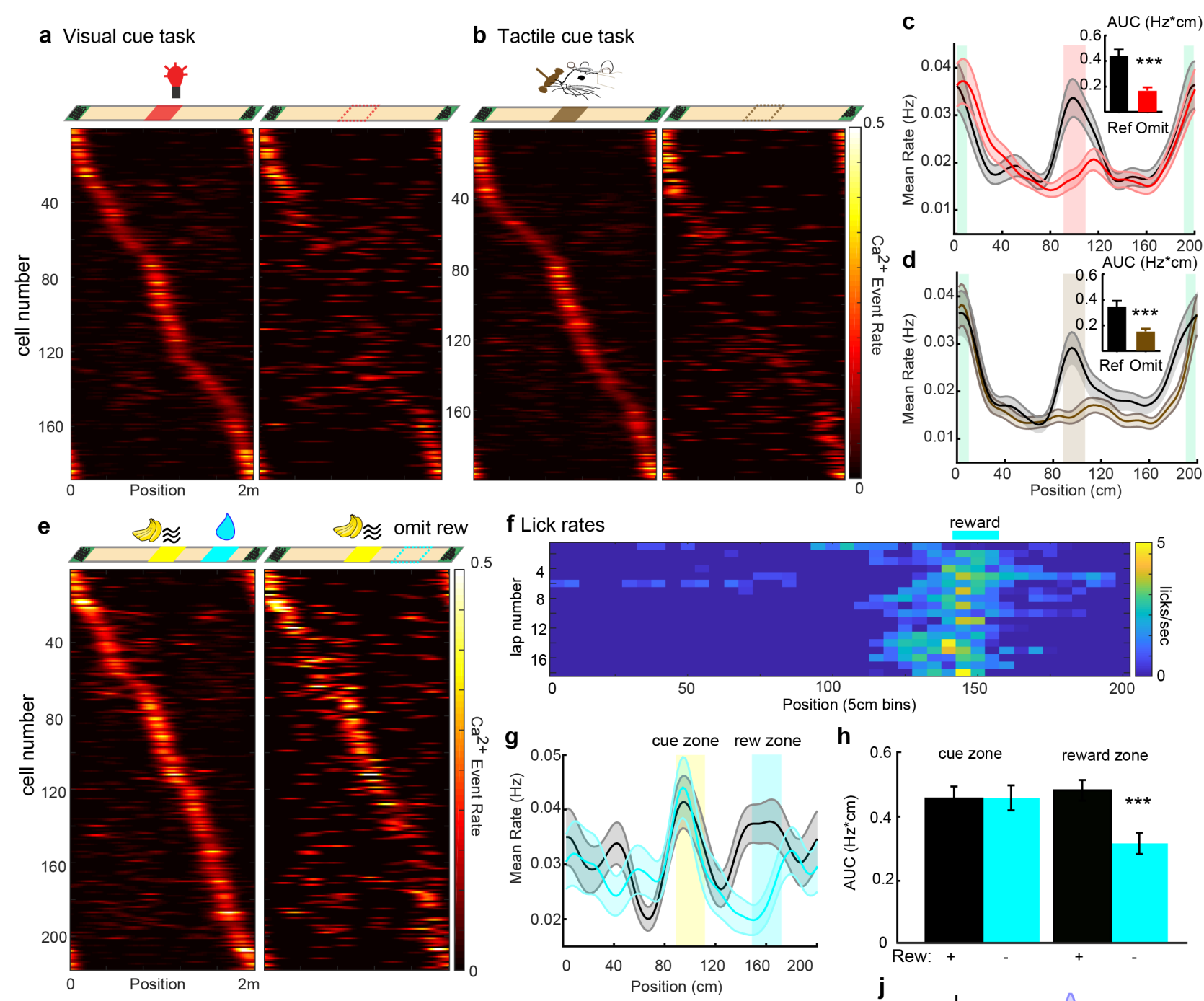

i Three cue task
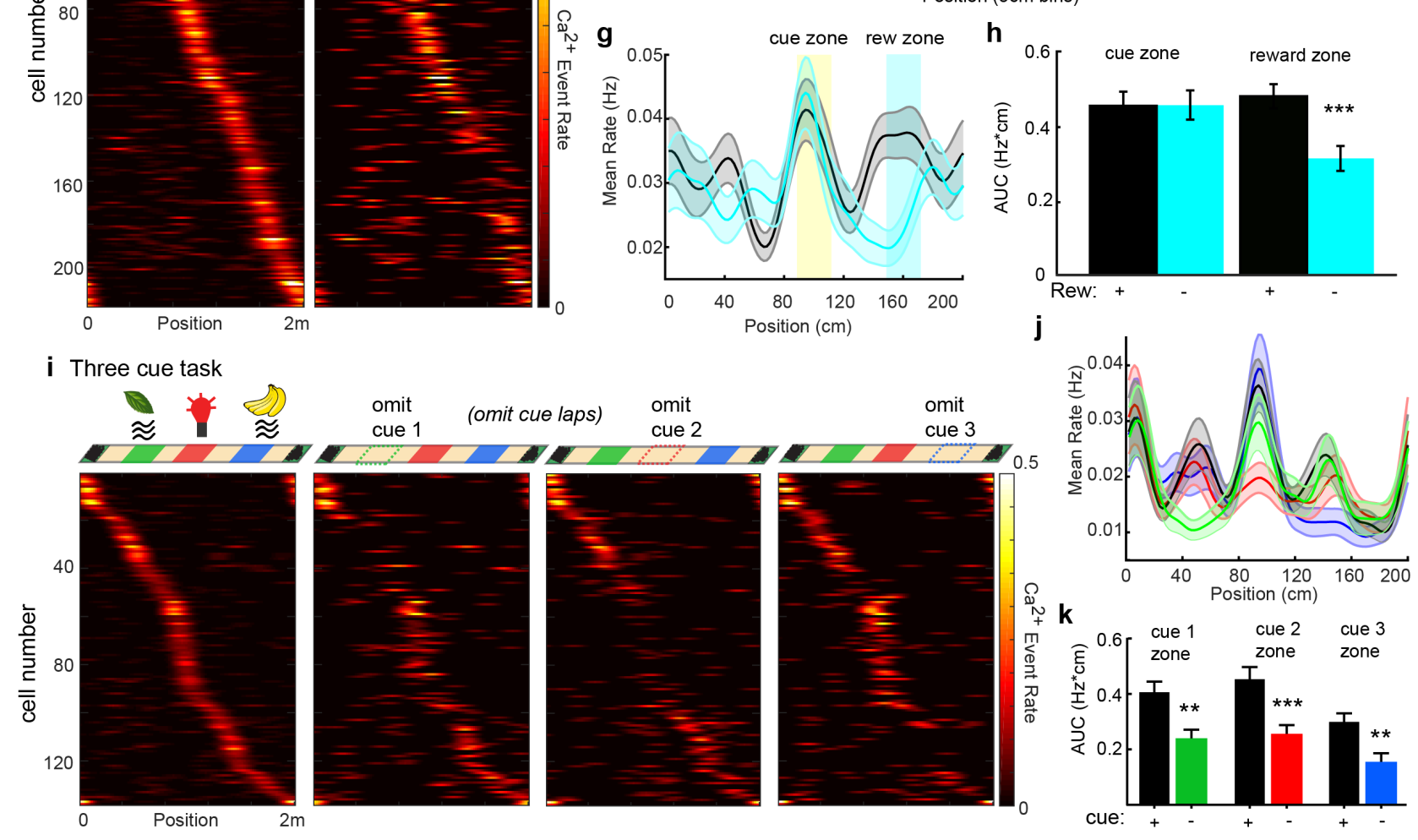

j

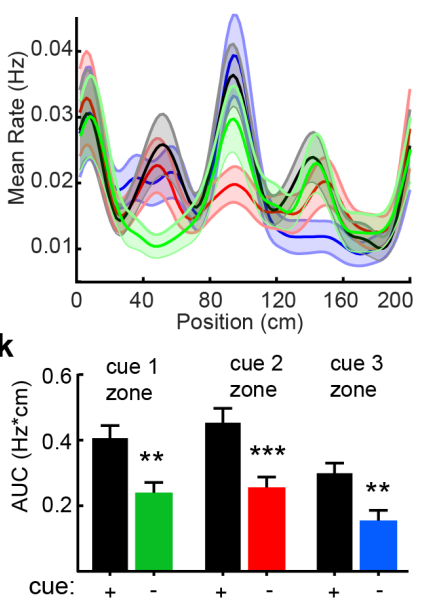

Supplementary Figure 2. Single visual and tactile cues, reward and multiple salient cues evoke robust responses in DG.

a) Firing of DG neurons in response to an LED visual cue. Top: Location of lap cue (green boxes) and an LED cue (red box), during normal and cue-omitted trials. Bottom: spatial firing rates of 188 
spatially tuned neurons ( $n=6$ mice) during the first exposure to the middle visual cue on normal (left), and cue-omitted (right) laps.

b) Firing of DG neurons in response to a whisker tactile cue. Top: Location of lap cue (green boxes) and a whisker tactile cue (brown box), during normal and omit trials. Bottom: spatial firing rates of 199 spatially tuned neurons ( $n=8$ mice) during the first exposure to the middle tactile cue on normal (left), and cue-omitted (right) laps. Each row represents a cell, and the $x$ axis represents the treadmill position.

c) Average spatial firing rate for neurons shown in "e" (LED cue) on normal (black, mean \pm SEM), and cue-omitted (blue) laps. Inset shows the averaged area under the firing rate curves $\left(\mathrm{Hz}^{*} \mathrm{~cm}\right)$ within the visual cue region during normal laps (black bar) compared to the same region during cue omitted laps ( $p<0.0001$, Wilcoxon Signed Rank Sum test).

d) Average firing rates of neurons shown in " $\mathrm{f}$ " (tactile cue) on normal (black), and cue-omitted (blue) laps. Inset shows the averaged area under the firing rate curves $\left(\mathrm{Hz}^{*} \mathrm{~cm}\right)$ within the tactile cue region during normal laps (black bar representing blue area under the middle region of the treadmill) compared to the same region during cue omitted laps $(p<0.0001$, Wilcoxon Signed Rank Sum test).

e) Top: Behavioral task with a static operant reward at $75 \%$ track length, preceded by an olfactory cue in middle of track. Bottom: Spatial firing rates for all spatially tuned cells in the static reward task. Laps with olfactory cue in middle of track and reward at $75 \%$ track length (static rew., left), or laps with reward omitted (omit rew., right). ( $\mathrm{n}=256$ cells, 4 mice, 7 sessions).

f) Averaged spatial lick rates over laps, for behavioral sessions in "e".

g) Average spatial firing rates over all cells in "e" during static rew. laps (blue), and omit rew. laps (red). Olfactory cue zone shaded in yellow, reward zone shaded in orange.

h) Averaged area under the firing rate curves for cue zone and reward zone on static reward and omit reward laps. $p=3.8 \mathrm{e}^{-9}$, reward zone rate during rew+ vs. rew- (omit rew.) laps, Wilcoxon signed rank test.

i) (Top, left) Experimental setup with 3 cues, cue 1 = mint odor (olfactory), cue 2 = LED (visual), cue 3 = isoamyl acetate odor (olfactory). Individual cues are omitted on intermittent laps (right). (Bottom) Spatial calcium event rates for the same tuned cells in normal 3-cue laps (left), and intermittent laps where individual cues are omitted (right 3 cols., as labeled, n=138 cells from 5 mice, 1 session/mouse, sorted by normal 3-cue lap activity).

j) Mean calcium event rates over all cells in "e" on normal laps (black), laps where cue 1 is omitted (green), cue 2 omitted (red), or cue 3 omitted (blue).

k) Averaged area under the firing rate curves within zones corresponding to cues 1-3 on normal laps (black), laps where cue 1 is omitted (green, $p=0.0031$ ), cue 2 omitted (red, $p<0.001$ ), or cue 3 omitted (green, $p=0.0047$ ). Wilcoxon matched-pairs signed rank test, error bars are mean \pm SEM. 

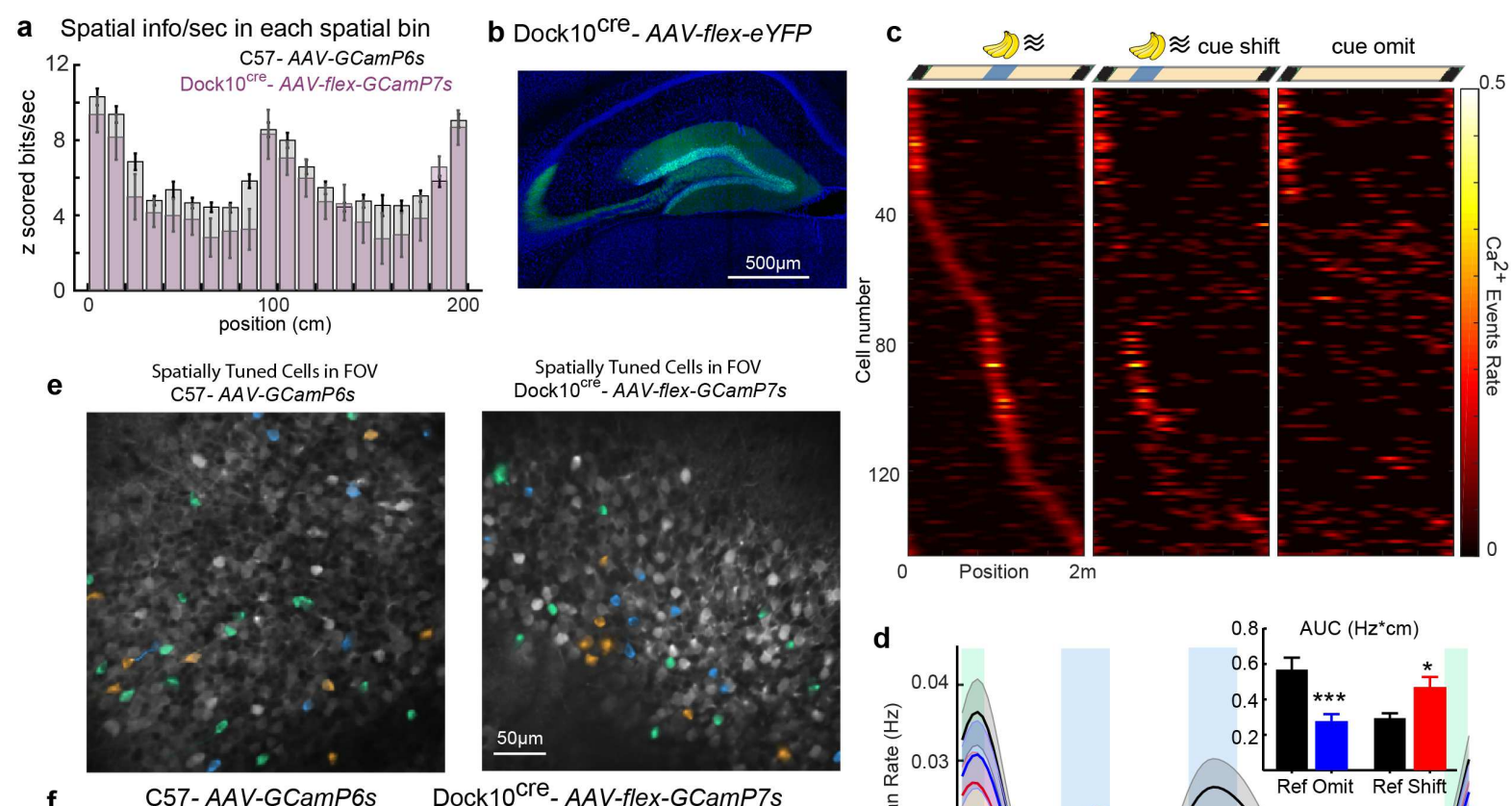

$\mathbf{f}$

C57- AAV-GCamP6s

Dock10 $10^{\text {Cre }}$ - AAV-flex-GCamP7s
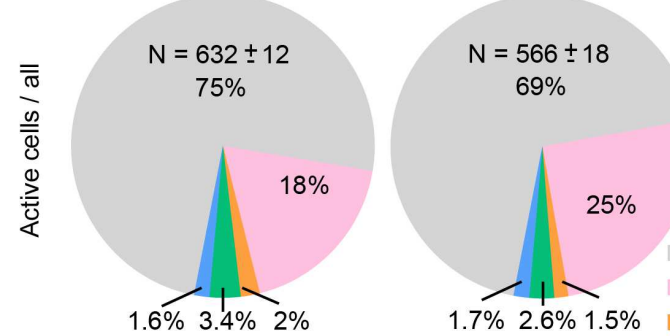

Inactive cells $1.7 \% 2.6 \% 1.5 \%$
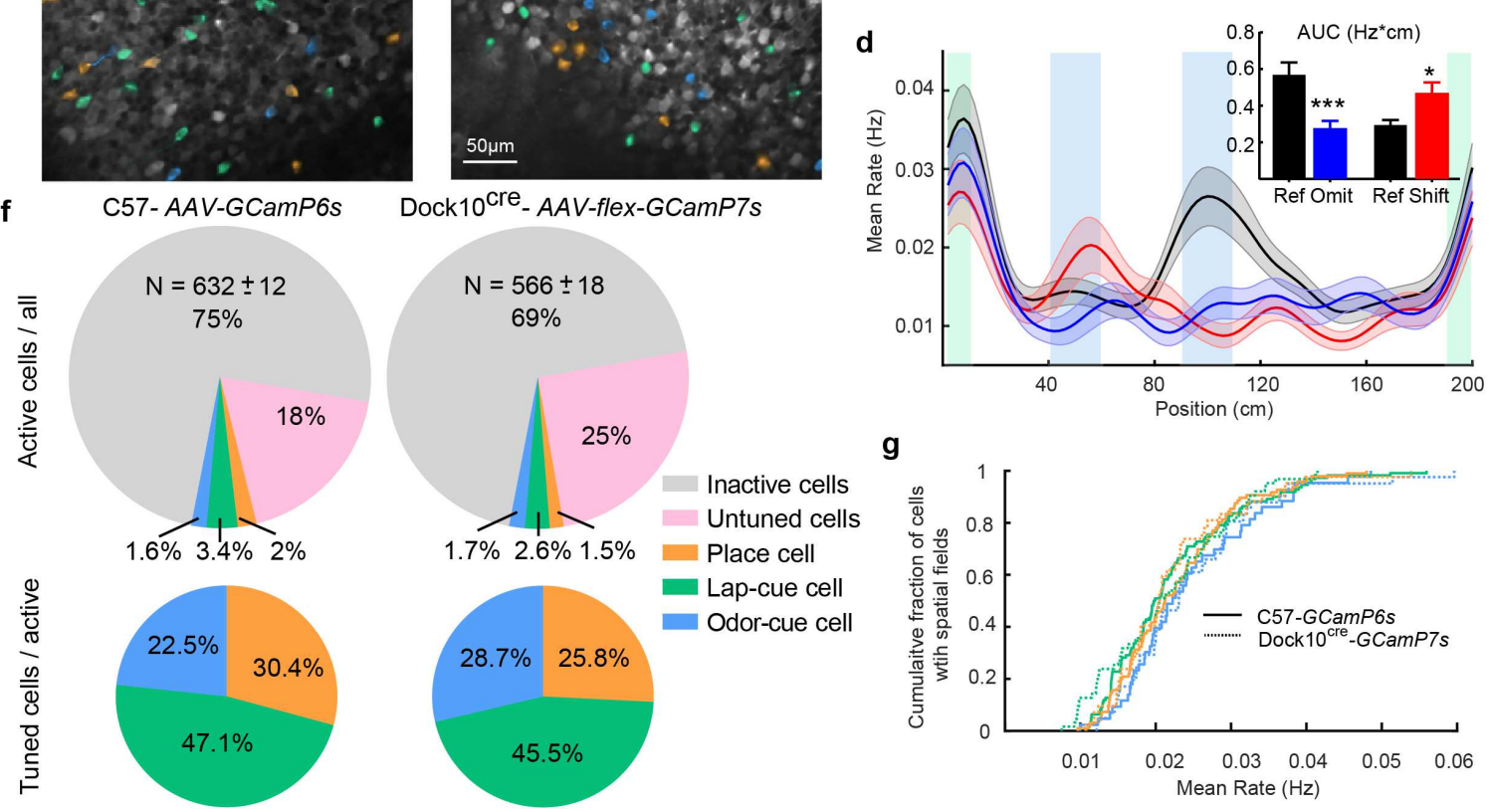

g

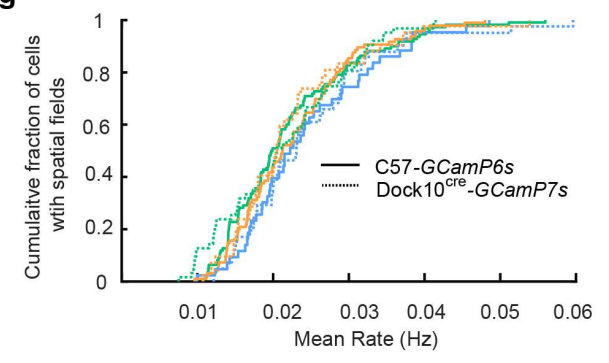

Supplementary Figure 3. Representations of sensory cues and place in a granule cell specific transgenic mouse, Dock10-cre

a) Mean spatial information by position. Average $Z$ scored spatial information for cells binned by tuning position during on-line cue manipulation task. WT dorsal dentate gyrus neurons express GCamP6s (gray bars) and Dock10-cre labelled granule cells express FLEX-GcamP7s (purple bars). Error bars represent SEM, $P_{s p k z-D G-s p k z-G C}=0.15$, Wilcoxon rank-sum test calculated over cumulative mean spkZ.

b) Confocal image projection of brain slice from Dock10-cre mouse injected with FLEX-eYFP AAV in the dorsal dentate gyrus (blue=DAPI, green=eYFP).

c) Spatial firing rates of Dock10/GCaMP7s-expressing granule cells in treadmill task with an olfactory cue. (Left) normal middle cue responses, (middle) cue shift laps, (right) laps in which odor cue is omitted ( $n=146$ cells from 4 sessions in 2 mice). Cells sorted by activity on normal middle cue laps

d) Average spatial firing rates from all cells in "c" during normal middle cue (black), cue shift (red), and cue omitted laps (blue). (Inset) averaged area under the firing rate curves $\left(\mathrm{Hz}^{*} \mathrm{Cm}\right)$ within the 
middle cue region during normal (black bar), cue omitted (blue bar), and cue shifted laps (red bar). $P_{\text {Normal-Omit }}<0.001, P_{\text {Normal-Shift }}=0.03$, Wilcoxon matched-pairs signed rank test, error bars are mean \pm SEM.

e) A representative in vivo two-photon field of view within the dentate gyrus of C57 mice expressing GCaMP6s (left) and Dock10-cre mouse expressing FLEX-GCaMP7s in granule cells (right), including spatially scattered odor (blue) and lap (green) cue cells and place cells (orange). f) Fraction of inactive cells (gray), active cells (with at least 0.001 transients per $s$, pink) and spatially tuned cells (orange, green, blue). Mean ${ }_{C 57-G \text { camP6s }}=631.68 \pm 11.9$ cells, from 8 mice 2 sessions each, Mean Dock10Cre-GcamP7s $=566.11 \pm 18.4$ cells, mice/session from 2 mice 2 sessions each. Small, round somata corresponding to granule cells within the granule cell layer were counted using the Cellpose algorithm followed by manual inspection in a subset of total sessions imaged from one mouse.

g) Cumulative distribution curve of the firing rate of odor cue, lap cue and place cells in Dock10cre mice; Odor Cue $P_{\text {C57-GcamP6s-Dock10Cre-GcamP7s }}=0.11$, Lap Cue $P_{\text {C57-GcamP6s-Dock10Cre-GcamP7s }}=0.05$, Place cell $P_{C 57-G c a m P 6 s-D o c k 10 C r e-G c a m P 7 s}=0.05$, Comparisons are Wilcoxon rank-sum test. 
a All spatially tuned cells

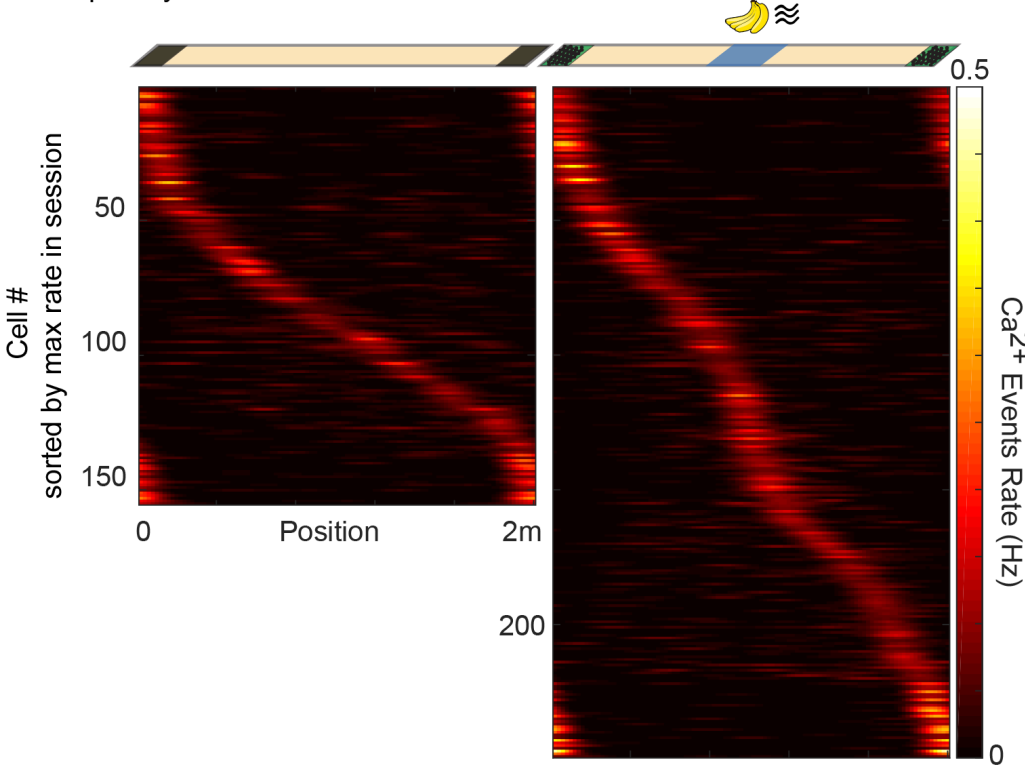

e Registered Odor cue cells

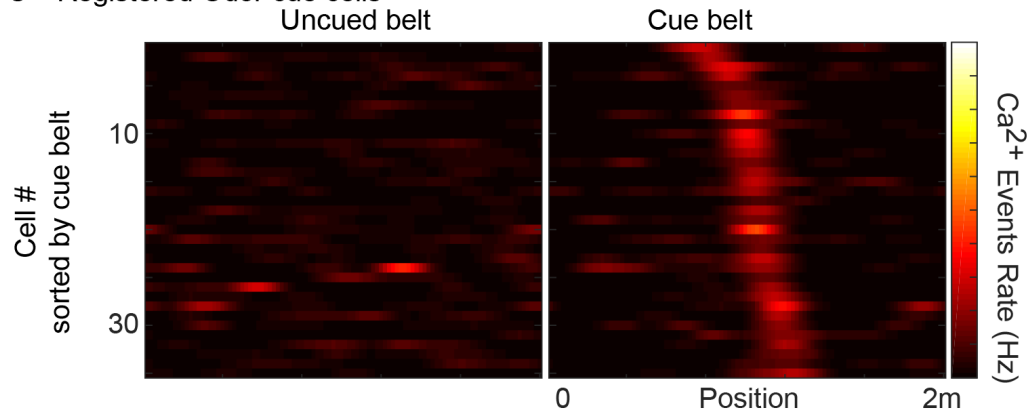

b Fraction of all spatially tuned cells

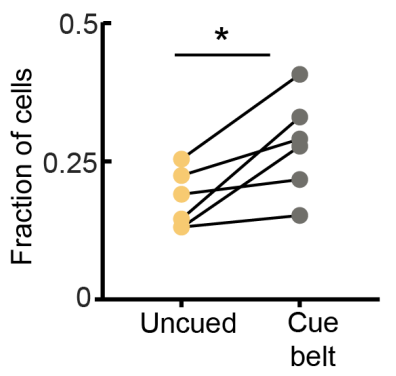

c Spatial Information

(excluding odor cue cells)

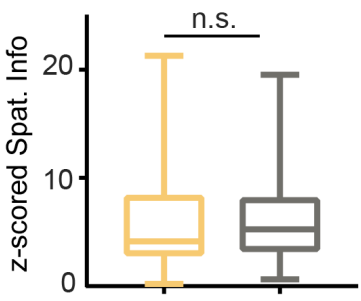

d Tuning Consistency (excluding odor cue cells)

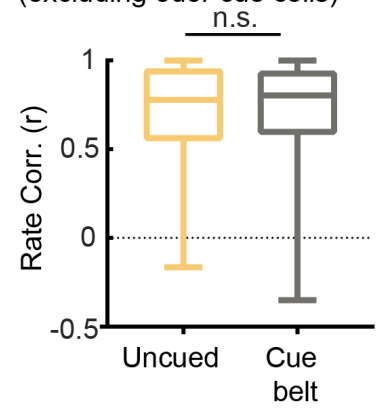

f Fraction of registered cells

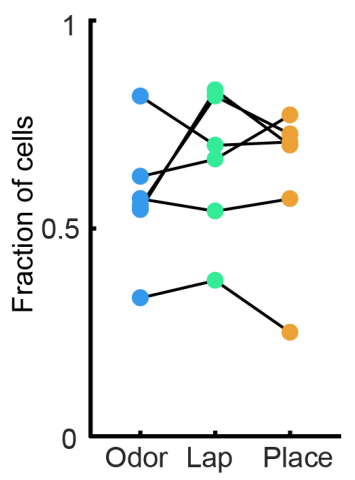

g Fraction cells tuned in both belts

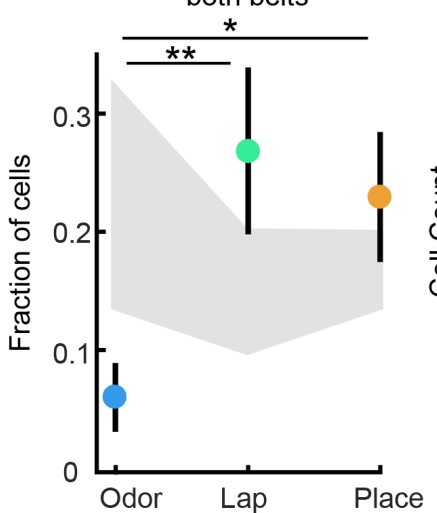

h Odor-cue Cells Lap-cue Cells Place Cells

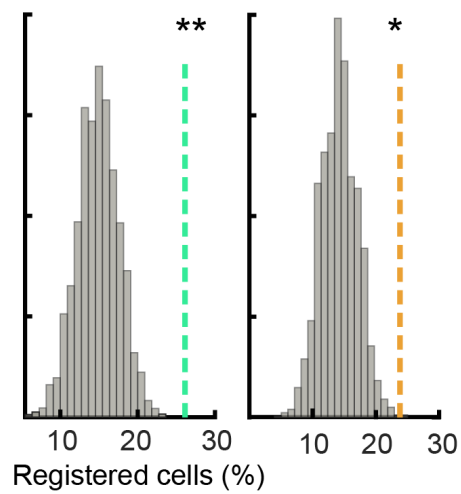

Supplementary Figure 4. Odor responsive cells are not spatially tuned on an uncued belt. a-c) Comparison of cell tuning properties between uncued vs. cued treadmill belts. a) Spatial firing rates of all tuned DG neurons within the same fields of view (FOV) recorded during paired sessions on an uncued belt (left) and odor cued belt (right). Neurons are ordered according to the 
position of their peak rate in each belt (uncued: 155, cue: 249 spatially tuned neurons in 6 mice, 1 session/mouse), and are not matched between uncued and cue belts.

b) Fraction of active cells that are spatially tuned (with at least 0.001 transients per $s$ ) in matched FOVs recorded on uncued and cue belts ( $p=0.0313$, Wilcoxon signed-rank test, $n=6)$.

c) $Z$ scored spatial information of all spatially tuned neurons in uncued $(n=155)$ and cued sessions $(n=193)$, excluding odor-cue responsive neurons ( $p=0.0897$, Wilcoxon rank-sum test).

d) Tuning consistency (firing rate correlation between first and last halves of the session) of all spatially tuned neurons in uncued and cued sessions, excluding odor-cue responsive neurons $(p=0.5237$, Wilcoxon rank-sum test). Boxes, 25th to 75th percentiles; bars, median; whiskers, $99 \%$ range.

e-h) Comparison of registered cue and place cell responses between uncued and cue belts. e) Activity of cross-registered odor cue cells in uncued and cue belts. Cells were classified as odorcue cells in cue belt and then their responses were determined in uncued belt. Note that these cue cells were active but not spatially tuned in uncued sessions.

f) Fraction of odor-cue (blue), lap-cue (green) and place cells (orange) that are cross- registered in uncued belt sessions, and are therefore active but not necessarily spatially tuned $(p=0.838$, $\mathrm{n}=6$ matched sessions, Friedman's test).

g) Fraction of registered cells that are spatially tuned in uncued belt sessions and encoded the odor cue (blue), lap cue (green), or place cells (orange) on the cued belt $\left(x^{2}=10.88, p=0.0043\right.$, POdorCue-LapCue $=0.0066$, POdorCue-Place $\left.=0.0286, P_{\text {LapCue-Place }}=0.9076\right)$. Gray area represent $2.5^{\text {th }}$ and $97.5^{\text {th }} \%$ of null distributions for each cell type. Error bars, mean \pm SEM.

h) The fraction of cross-registered odor cue cells that were spatially tuned on the uncued belt is significantly below the null distribution (left) while the fraction of lap-cue (middle) and place cells that are spatially tuned remain significantly above the null distribution. The null distributions are generated for each cell type by randomly permutating cell IDs of all cross-registered neurons and determining overlap among cell types. (Level of significance for 5,000 shufflings ${ }^{* *} p<0.01$; ${ }^{*} p<$ $0.05, N_{\text {Odor-cue }}=35, N_{\text {Lap-cue }}=54, N_{\text {Place }}=58$ ). 

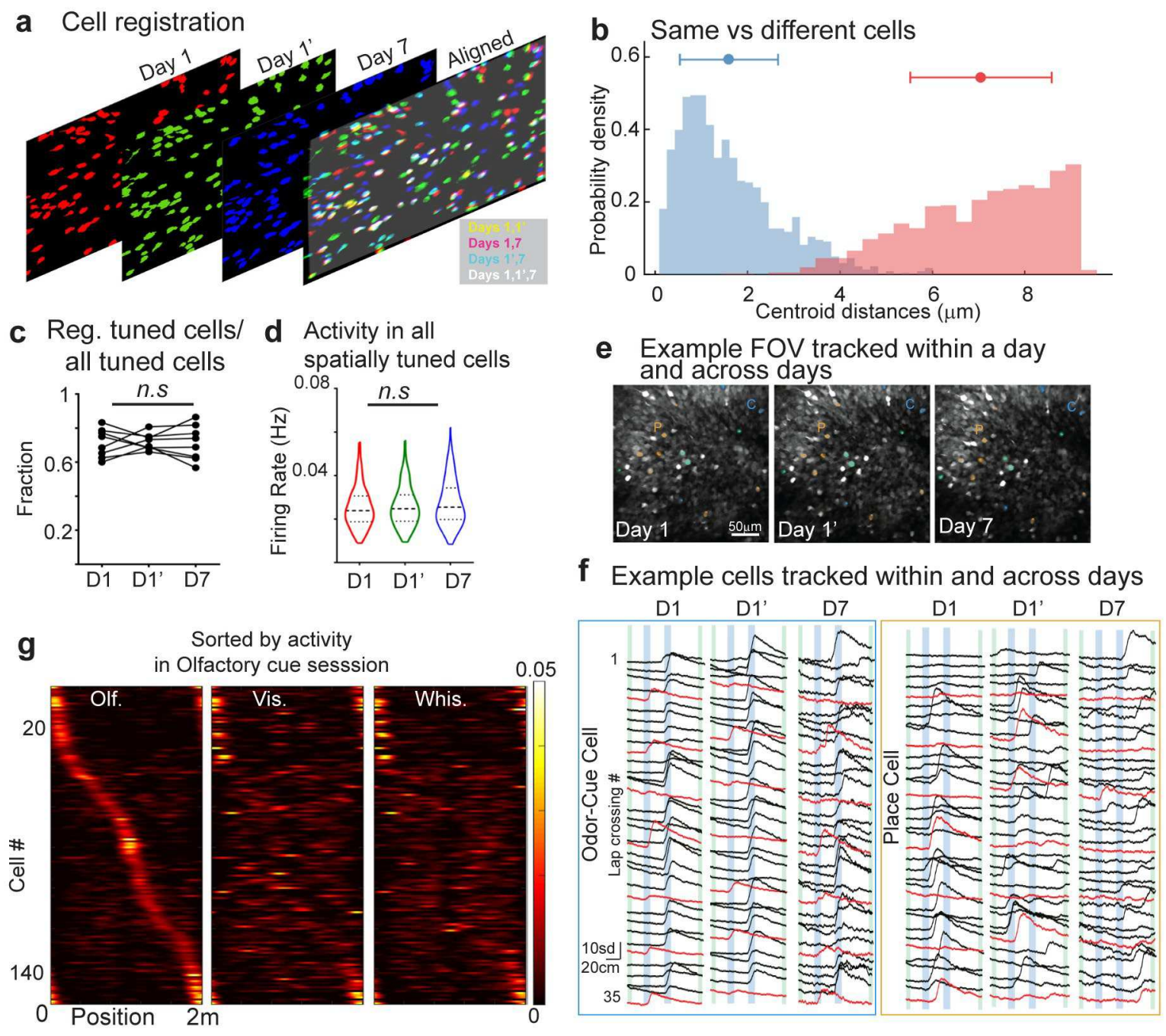

\section{Supplementary Figure 5. Multisession tracking of individual cue and place cells}

a) Representative alignment of spatial footprints for cells segmented across sessions within a day or over $1 \mathrm{wk}$. in a single imaging field.

b) Distribution of centroid distances between registered (blue, $1.45 \pm 0.97, n=1604$ ) and nonregistered (pink, $7.07 \pm 1.5, n=2214$ ) neighboring cell pairs ( $p=6.910^{-43}$, Wilcoxon Rank Sum test). c) Fraction of all spatially tuned cells in day 1 session1 (D1), day 1 session 2 (D1') and day 7 (D7) that are registered to at least one other session, $x^{2}=0.25, \mathrm{p}=0.9674, P_{D 1-D 1}>0.9999, P_{D 1}$ $D 7>0.9999, P_{D 1}{ }^{\prime}-D 7>0.9999, \mathrm{n}=8$ matched sessions, Friedman and Dunn's multiple comparisons tests.

d) Comparison of average firing rates in all tuned cells across days regardless of tracking $X^{2}=4.993, p=0.0824, P_{D 1-D 1}>0.9999, P_{D 1-D 7}=0.0781, P_{D 1^{\prime}-D 7}=0.4789, N_{D 1}=417, N_{D 1}{ }^{\prime}=365$, $N_{D 7}=338$, from 8 matched sessions, Kruskal Wallis and Dunn's multiple comparisons tests .

e) Representative fields of view with odor cue cells (blue), lap cue cells (green), and place cells (orange) tracked within a day and across days.

f) Representative $\mathrm{Ca}^{2+}$ transients for an odor cue cell that has stable cue-selective activity within one day and over $1 \mathrm{wk}$ (left). Transients for place cells show relatively stable firing location within 
day but a reorganization of spatial selectivity across days (right). Black and red traces represent normal and cue shifted laps, respectively.

g) Firing rates of tracked neurons in visual and whisker tactile cue sessions ordered according to the position of peak activity in olfactory cue sessions ( $n=157$ cells, 6 mice). 
Dual cue location task

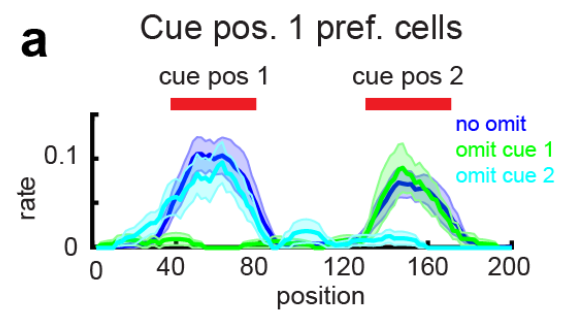

C Cue pos. 1 pref. cells

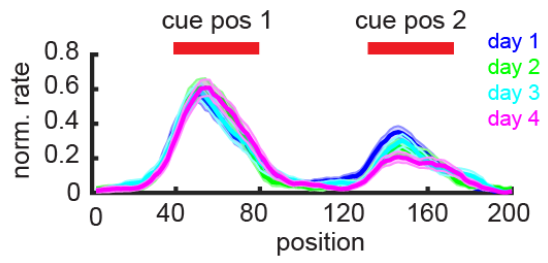

Cue pos. 2 pref. cells

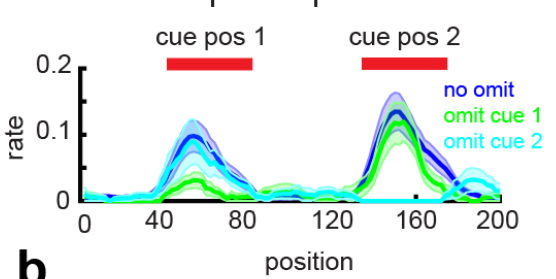

Cue pos. 2 pref. cells
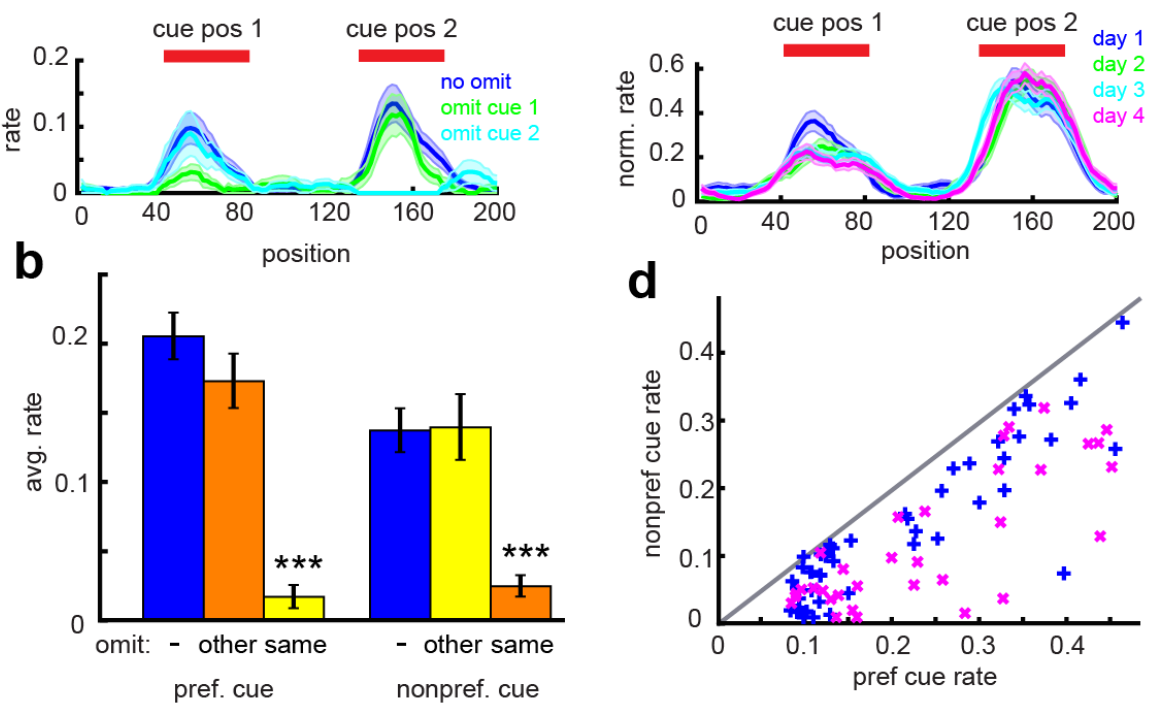

\section{Supplementary Figure 6. Spatial modulation of sensory cue responses}

a) Dual cue location task: Average spatial firing rates for cue cells preferring cue position 1 (top) and cue position 2 (bottom) on normal laps (blue), laps where the cue at position 1 is omitted (green), and laps where the cue at position 2 is omitted (cyan). ( $n=89$ pos 1 cells, 67 pos 2 cue cells).

b) Average peak firing rates for all cue cells (both cue 1 and cue 2 preferring) for preferred cue location (left) and non-preferred location (right) on normal laps (blue), laps where the opposite cue is omitted (orange), and laps where the same cue (preferred or non-preferred) is omitted. $(\mathrm{n}=156$ cells, positions $1 \& 2)$ (mean \pm SEM: $0.205 \pm 0.017,0.173 \pm 0.020,0.017 \pm 0.008,0.137 \pm$ $0.016,0.140 \pm 0.024,0.025 \pm 0.008$, P Preferred Cue $=7.16 \times 10^{-9}, P_{\text {Non-preferred Cue }}=3.48 \times 10^{-9}$, Wilcoxon sign rank test).

c) Average spatial firing rates for cue cells preferring cue position 1 (top) and cue position 2 (bottom) on days 1 (blue, $n=50$ ), 2 (green, $n=40$ ), 3 (cyan, $n=50$ ), and 4 (magenta, $n=33$ ) of dual cue location task.

d) Cue location firing rates for preferred and non-preferred cue location for all cue cells on days 1 (blue, $n=50$ ) and 4 (magenta, $n=33$ ) of dual cue location task. 
a Population correlation during normal and cue omitted laps

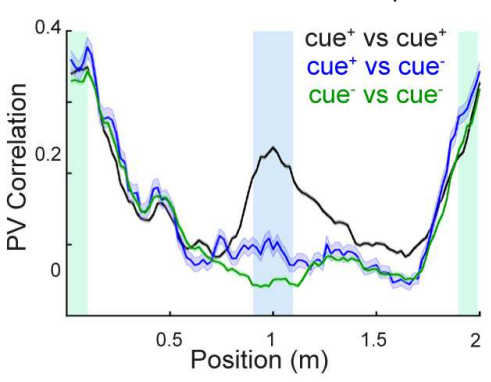

d Decoder error in normal vs cue omitted laps

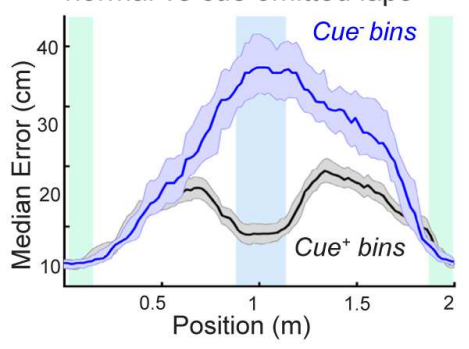

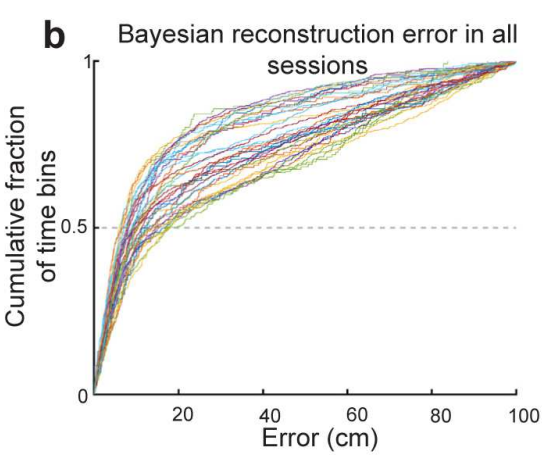
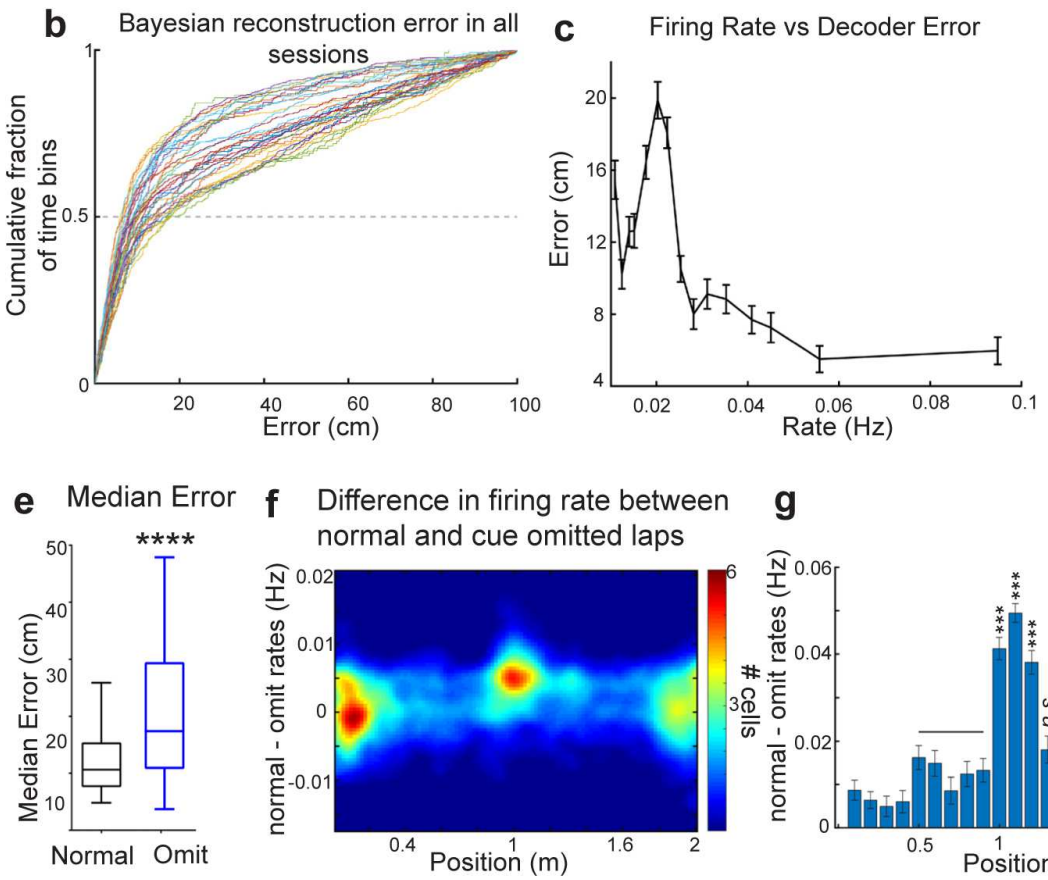

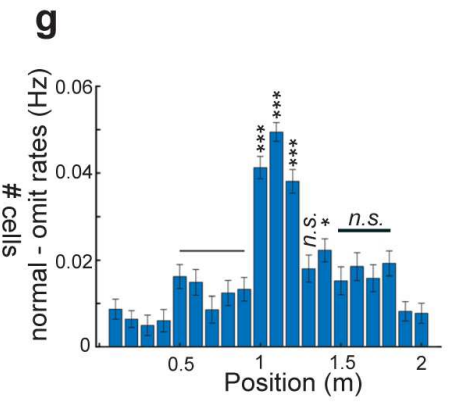

Supplementary Figure 7. Effect of sensory cues on spatial coding during cue-omitted laps

a) Population vector correlations for all spatially tuned cells at each treadmill position for normal middle cue laps (black) and omitted cue laps (blue). Locations of the middle and lap cue are shown blue and green shaded areas, respectively.

b) Bayesian decoding: Cumulative distribution functions of the error of the maximum likelihood estimate of position for 30 example recording sessions.

c) The decoder error for spatially tuned neurons plotted against their firing rate.

d) Decoding error for each treadmill position in normal (black) and cue omitted laps (blue). Shaded error region represents the difference of the median from the null distribution of median decoder error.

e) Average decoding error for normal and cue omitted laps calculated as the absolute median distance between the decoded value in each time bin and the actual value of the position. $p<0.0001$, Wilcoxon signed-rank test ( $n=66$ sessions). Boxes, 25th to 75th percentiles; bars, median; whiskers, $99 \%$ range.

f) Effects of cue omission on individual cells: Color histogram of all spatially tuned cells, plotted based upon spatial field location and difference in firing rate between normal cue and cueomitted laps.

g) Average difference in normal middle cue and cue-omitted lap firing rates based upon cell spatial field location. Note that the largest cue effects are confined to cells immediately responsive to the middle cue. With respect to pre-cue cells, Wilcoxon Rank Sum test: ${ }^{* *}=p<$ $10 \mathrm{e}-10,{ }^{*}=\mathrm{p}=0.03$, n.s. $=$ not significant. 
a

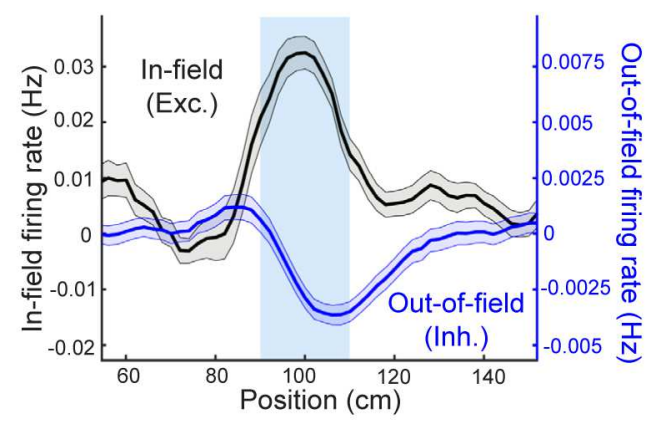

b

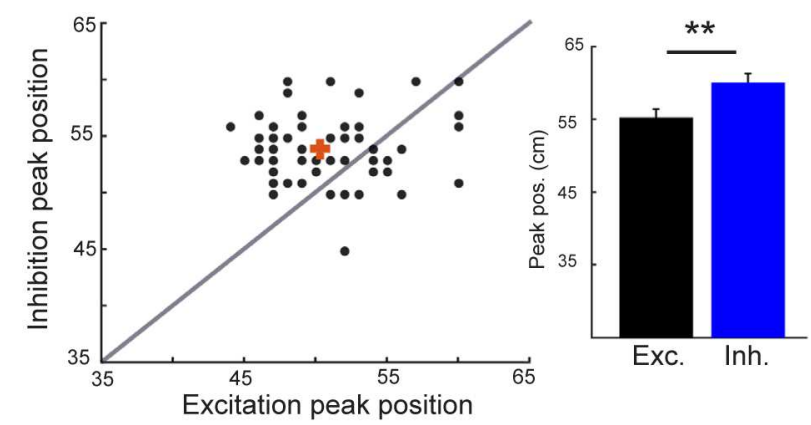

\section{Supplementary Figure 8. Timing of cue-related inhibition in the DG}

a) Average in-field spatial firing rates (i.e. cue-related excitation) in the region around the middle cue location for all tuned neurons, compared with out-of-field firing rates (i.e. cue-related inhibition), and adjusted for pre-cue firing rate. The position of the excitation peak precedes the nadir of inhibition. Blue shaded area shows the cue delivery position.

b) (Left) Session averaged excitatory (within-field) peak position vs. inhibitory (out-of-field) peak position (66 sessions). Red = avg., diagonal (gray line). (Right) Quantification of the position of excitation peak compared to the position of inhibition peak, $p<0.01$, Wilcoxon Signed Rank Sum test. 

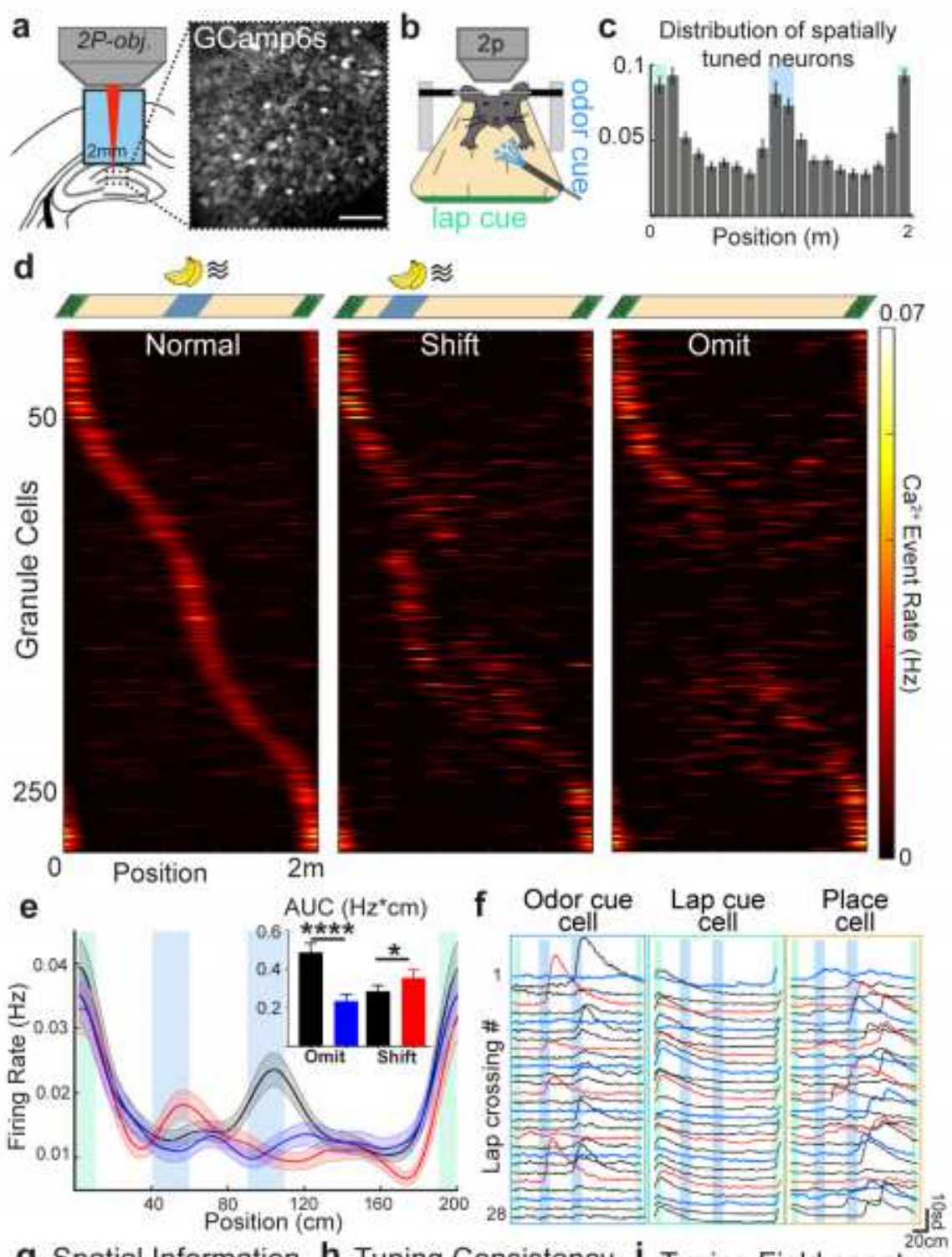

g Spatial Information $\mathbf{h}$ Tuning Consistency $\mathbf{i}$ Tuning Field onset
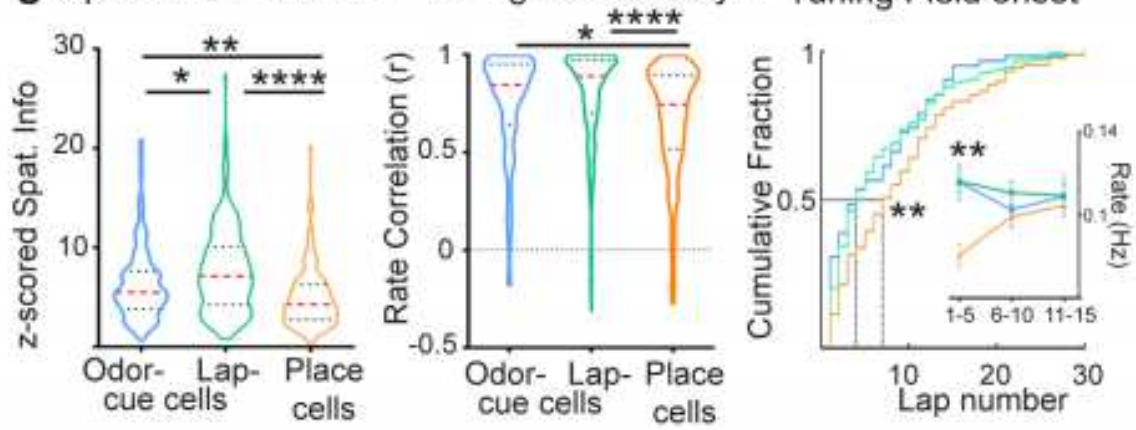

\section{Figure 1}

Robust representation of sensory cues in the dentate gyrus a) Two-photon imaging of DG population calcium activity. Left, diagram of the imaging window implant in the dorsal dentate gyrus. Right, Time averaged in vivo two-photon image of GCaMP6sexpressing granule cells. b) Diagram of head-fixed 
treadmill apparatus for spatial sensory cue delivery. 2 c) Fraction of spatially tuned cells at each linearized treadmill position (bins $=10 \mathrm{~cm}$ ) per session, $n=4091$ cells from 8 mice across 6-9 sessions/mouse, locations of the odor and lap cue are shown blue and green shaded areas, respectively. d) Spatial patterns of DG neuron activity during cue task. Top: Location of lap cue (green boxes) and an odor cue (blue box), on normal, shift or omit laps. Bottom: Lap-averaged spatial firing rates of 285 spatially tuned neurons ( $\mathrm{n}=8$ mice) during their first session with the cue on normal middle location (left), cue-shifted (middle) and cue-omitted (right) laps. Each row across all graphs represents a single cell, and the $x$ axis represents the treadmill position. e) Average firing rates by position for neurons shown in panel D on normal (black), cue-shifted (red) and cue-omitted laps (blue, mean \pm SEM). (Inset) averaged area under the firing rate curves $\left(\mathrm{Hz}^{\star} \mathrm{cm}\right)$ within the middle cue region during normal (black bar), cue omitted (blue bar), and cue shifted laps (red bar). Pnormal-omit $<0.0001$, Pnormal-shift $=0.02$, Wilcoxon matchedpairs signed rank test, error bars are mean \pm SEM. f) Example fluorescence traces from odor-cue, lap-cue and place cells within a single session. Black, red, and blue traces represent normal, cue shifted and omitted laps, respectively. Scale bars are $\mathrm{cm}$ and standard deviation from each cell's baseline fluorescence. g) Z scored spatial information of cue and place cell populations. Z scored spatial information, $\chi 2=48.47, p<0.0001$, POdorCue-LapCue=0.0387, POdorCue-Place=0.0028, PLapCuePlace $<0.0001$. h) Tuning consistency of cue and place cells. Firing rate correlation between first and last halves of the session, $\chi 2=32.60, p<0.0001$, POdorCue-LapCue $=0.2224$, POdorCue-Place $=0.0117$, PLapCuePlace<0.0001. i) Emergence of cue and place responses. Cumulative distribution of spatial field onset lap in normal laps for cue and place cells, $\chi 2=9.29, p=0.0096$, POdorCue-LapCue $=0.8925$, POdorCuePlace $=0.0194$, PLapCue-Place $=0.0091$. (Inset) Spatial field firing rates of cue and place cells during - 1-5th, 6-10th and 11-15th laps, main effect of cell type: $F 2,1473=6.73, p=0.0012$; cell type $\times$ lap number interaction $F 4,1473=2.44, p=0.04$; main effect of lap number: $F 2,1473=0.28, p=0.7$; Lap 1-5th, POdorCue-LapCue=0.99, POdorCue-Place $=0.0011$, PLapCue-Place $<0.0001,2$-way ANOVA and Tukey's multiple comparisons test. NOdorCue $=114$, NLapCue $=220$, NPlaceCell=160, from 8 mice 2 sessions each. Red dotted lines in violin plots show median, black dotted lines show quartiles. Comparisons are Kruskal Wallis and Dunn's multiple comparisons tests unless otherwise noted. ${ }^{\star} P<0.05 ;{ }^{* \star} P<0.01$; ${ }^{\star \star *} P<0.001$. See also Supplementary Figures 1-4. 
a Stability over time

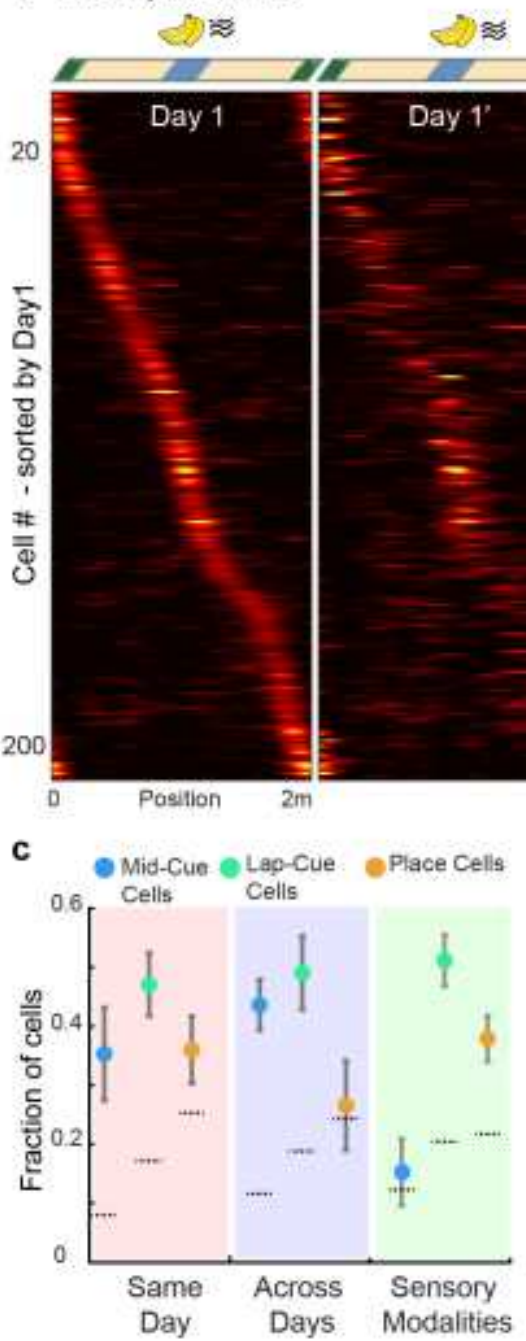

f Room context switch

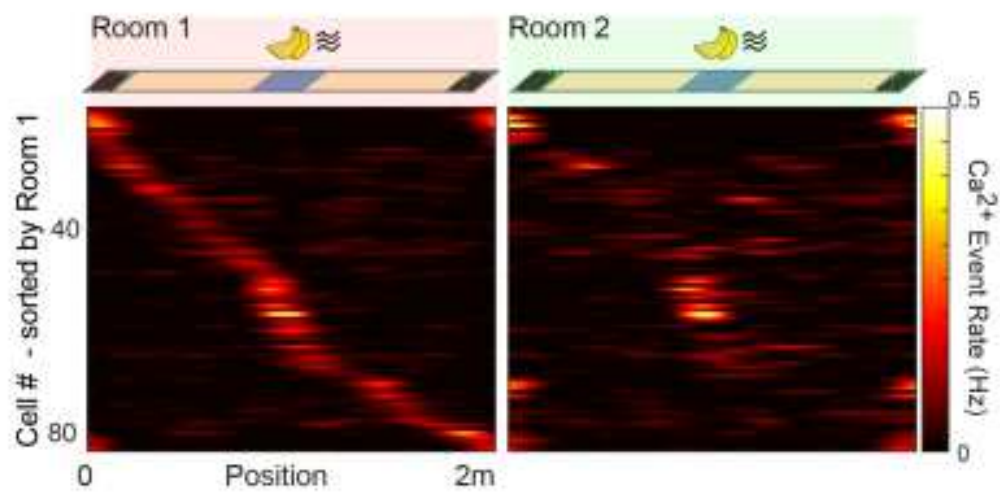

b Stimulus selectivity
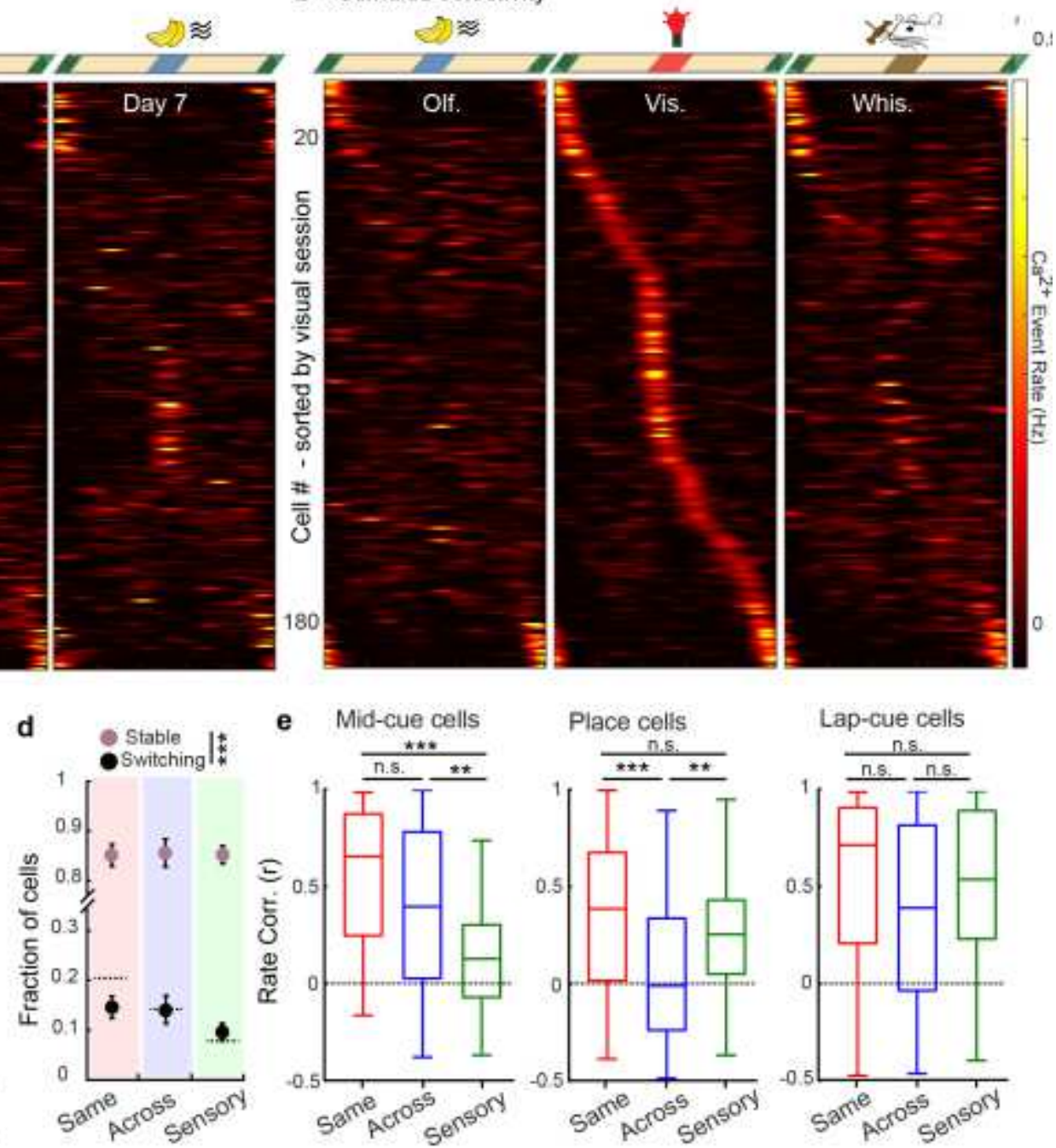

Lap-cue cells

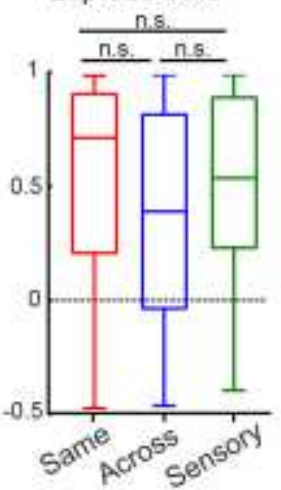

g

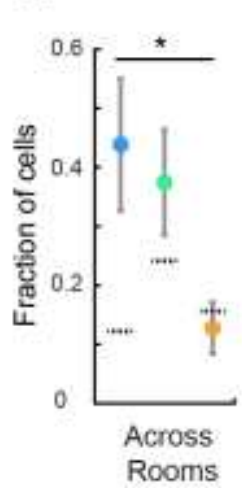

h

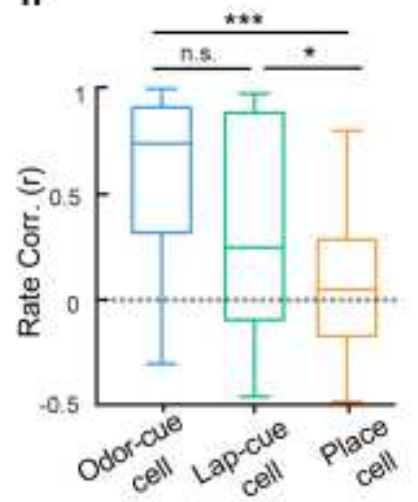

\section{Figure 2}

Sensory cue and place responses differ in stability and selectivity a) Spatial firing rates of individual DG granule cells matched between sessions within a day and over one week. Top: diagram of odor cue presentation during all sessions. Bottom: Spatial firing rates for spatially tuned neurons tracked during subsequent sessions on the same day or one week later, ordered according to the position of peak activity during the first exposure ( $n=233$ cells, 8 mice). b) Activity of granule cells in response to different sensory 
cues. Top: diagram of behavior in sessions with cues of different sensory modalities in the middle position and an invariant lap cue. 4 Bottom: Spatial tuning for neurons tracked through consecutive sessions during exposures to different cues. Data are shown for neurons with significant tuning in visual cue session and tracked in olfactory and whisker tactile cue sessions ( $n=196$ cells, 6 mice). c) Fraction of cross-session registered cells that encoded the same variable, lap cue (green), middle cue (blue), or place (orange), within same day (left), across days (middle), and during exposures to different sensory modalities (right) . Cells encoding the same variable on the same day, $\chi 2=1.81, p=0.4039$, PLapCueOdorCue $=0.4221$, PLapCue-Place $=0.549$, POdorCue-Place $=0.9713$; cells encoding the same variable across days, $\chi 2=14.36, p<0.001$, PLapCue-OdorCue $=0.1566$, PLapCuePlace $<0.0001$, POdorCuePlace $=0.0313$ (8 mice, 8 matched sessions, Day1-Day1', Day1-Day7); cells encoding the same variable with different sensory modalities, $\chi 2=19.78, p<0.00001$, PLapCueOdorCue $<0.00001$, PLapCuePlace $=0.0766$, POdorCue-Place $=0.0464$ ( 6 mice, 12 matched sessions, Odor-Vis. and Vis. - Tact.); KruskalWallis test, Dunn's multiple comparisons test. Dashed lines represent 97.5 th\% of null distributions for each cell type. Error bars, mean \pm SEM. d) Fractions of spatially tuned cells that stably encode only one variable (cue or place, pink) or switched response types (cue to place or vice versa, black) within same day, across days and in response to different sensory cues: PSwitch-Stable (Same Day) $<0.001$, PSwitchStable (Across Days) $<0.001$, PSwitch-Stable (Sensory Modalities) $<0.001$, Wilcoxon Rank Sum test. Dashed lines: 2.5th and 97.5 th\% of null distributions. Error bars, mean \pm SEM. e) Mean rate correlations within the same day (red), different days (blue) and different sensory cues (green) for mid-cue cells (left, $\chi 2=27.88, p<0.0001$, PSame Day-Across Days $=0.2465$, PSame DaySensory Modalities $<0.0001$, PAcross Days-Sensory Modalities=0.0038, NSame Day=37, NAcross Days=37, NSensory Modalities=55); place cells (middle, $\chi 2=18.79, p<0.0001$, PSame Day-Across Days $<0.0001$, PSame DaySensory Modalities $=0.7449$, PAcross Days-Sensory Modalities $=0.0055$, NSame Day $=76$, NAcross Days $=76$, NSensory Modalities=83), lap-cue cells (right, $\chi 2=5.096, p=0.0782$, PSame Day-Across Days $=0.0723$, PSame DaySensory Modalities $>0.9999$, PAcross Days-Sensory Modalities $=0.7932$, NSame Day $=80$, NAcross Days $=80$, NSensory Modalities=59). Kruskal-Wallis test, Dunn's multiple comparisons test. Boxes, 25th to 75th percentiles; bars, median; whiskers, 99\% range. ${ }^{\star} P<0.05$; ${ }^{\star \star} P<0.01$; ${ }^{* \star *} P<0.001$. f) Activity of DG granule cells in response to the same odor-cued track when recorded in different rooms. Top: diagram of exposures to an odor in the middle position and an invariant lap cue, performed in different rooms. Bottom: Spatial firing rates for tuned neurons tracked through consecutive sessions in different rooms ( $n=83$ cells, 4 mice). g) Fraction of cross-session registered cells that encoded the same variable, odor cue (blue), lap cue (green) or place (orange) in different recording rooms. $\chi 2=6.59$, $p=0.0372$, POdorCuePlace $=0.0490$, PLapCue-OdorCue $=0.9557$, PLapCue-Place $=0.0972$. Dashed lines: 2.5th and 97.5 th $\%$ of null distributions. Error bars, mean \pm SEM. h) Comparison of spatial firing rate correlations in all tuned cells across rooms $(\chi 2=15.85, p<0.001$, POdor-Lap cue $=0.27$, POdor Cue-Place $<0.001$, PLap Cue-Place $=0.03$, NOdor-Cue $=25$, NLapCue $=33$, NPlace=26). Kruskal-Wallis test, Dunn's multiple comparisons test. Boxes, 25th to 75th percentiles; bars, median; whiskers, $99 \%$ range. ${ }^{*} P<0.05$; $\star \star P<0.01 ; * * * P<0.001$. See also Supplementary Fig. 5 . 

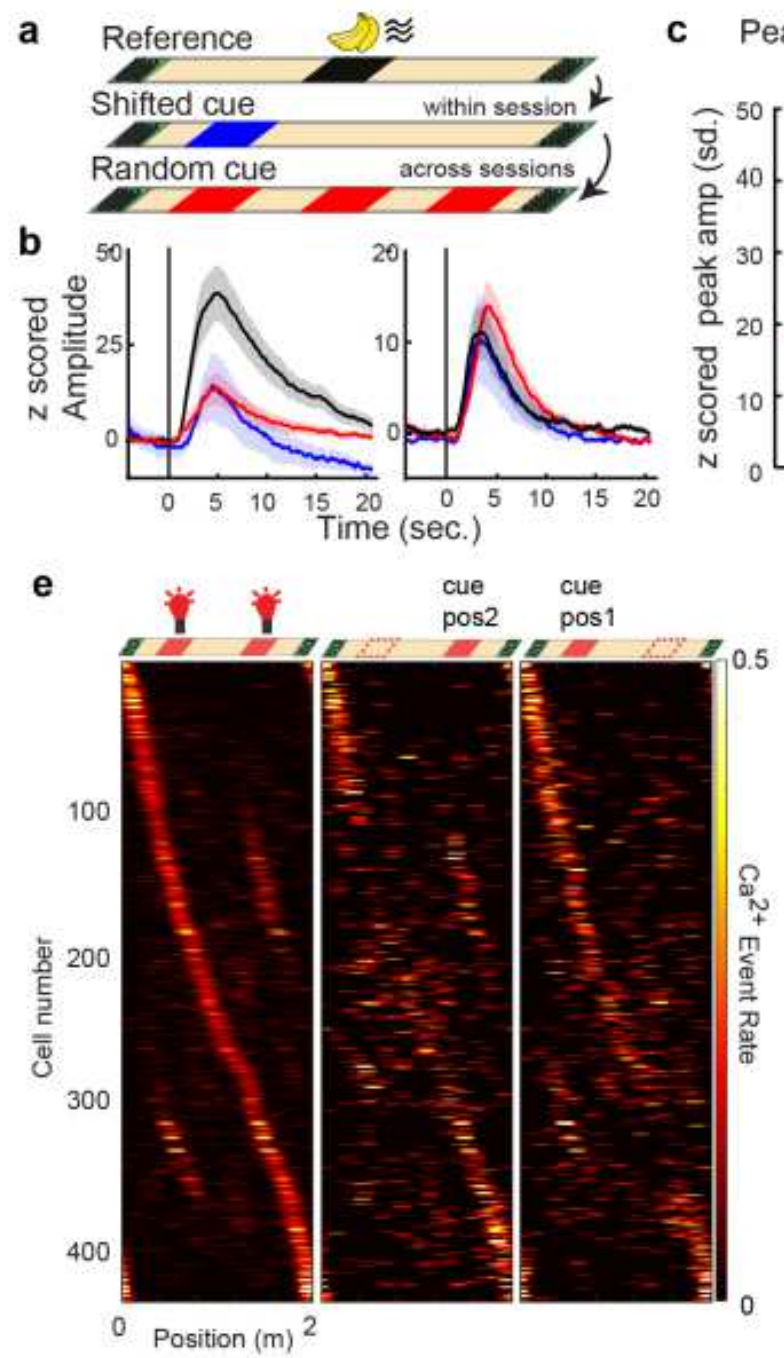

C Peak amplitude of cue reponses

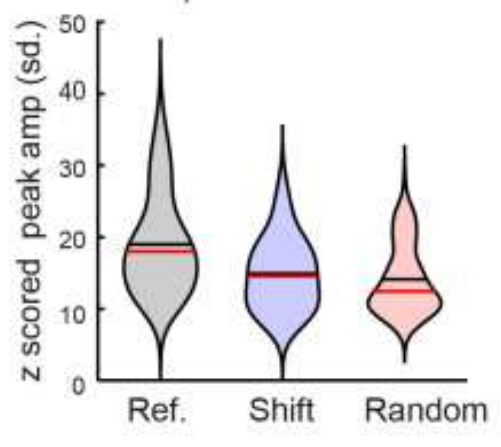

f Cue pos. 1 pref. cells

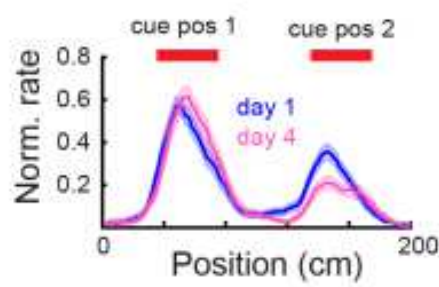

Cue pos. 2 pref. cells

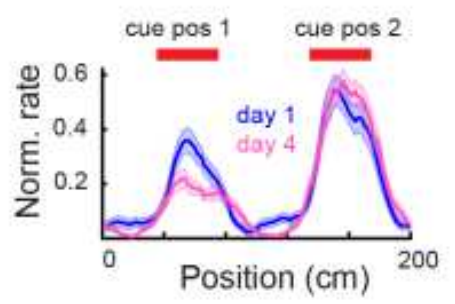

d Spatial modulation of cue reponses
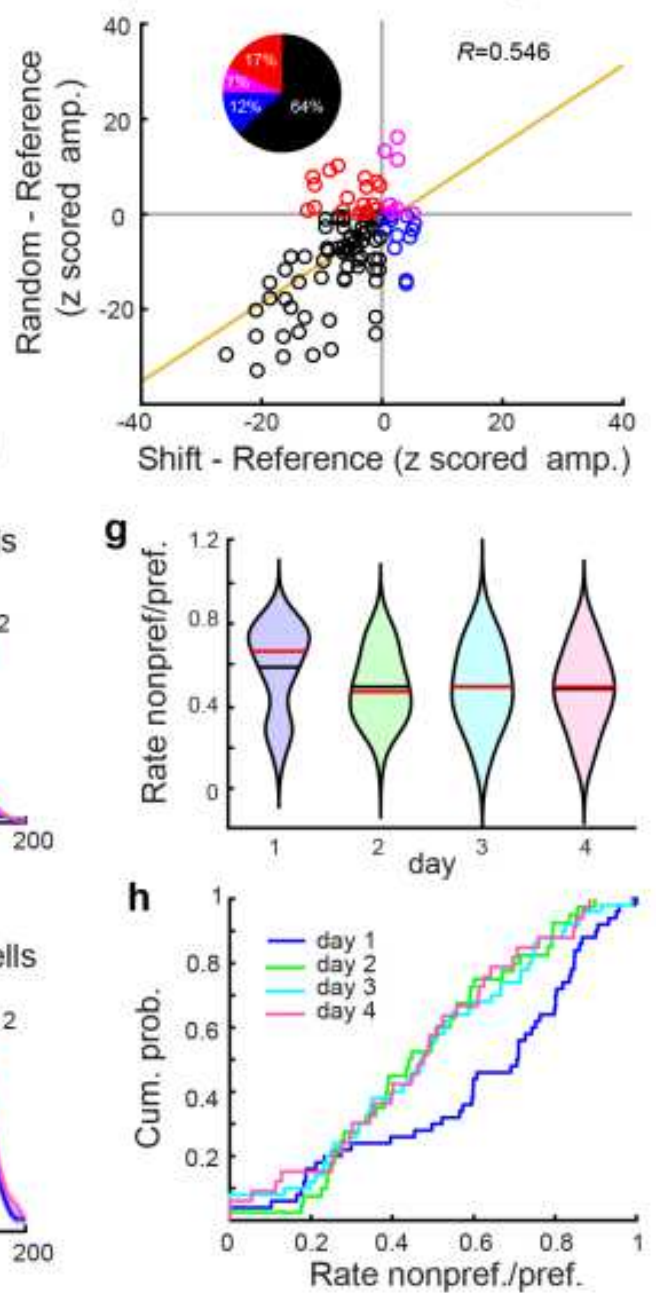

\section{Figure 3}

Sensory cue responses are spatially modulated a) Experimental setup to examine effects of cue-location pairing on cue responses. Cue responses are measured with respect to a typical middle cue location or an intermittently shifted cue location in the same sessions, or with random administration of the same cue on each lap in a subsequent session. b) Example cue-triggered average $\mathrm{Ca} 2+$ transients from two cue cells on normal (black), cueshifted (blue) and random-cue (pink) laps. Scale bars are sec and standard deviation from each cell's baseline fluorescence for the session. c) Z-scored peak amplitudes of Ca2+ transients from odor cue cells in normal, shift and random cue presentation conditions; $\chi 2=49.36$, $\mathrm{p}<0.001$, PNormal-Shift $<0.0001$, PNormal-Random<0.0001, PShiftRandom $>0.9999$, Friedman test and Dunn's multiple comparisons tests ( $n=101$ cells, 5 mice). Red lines in violin plots show median, black lines show mean. d) Difference in the magnitude of cue responses in normal laps from random-cue and cue-shift laps. Yellow line, linear regression $(R 2=0.546, p=3.5 \times 10-9)$. Pie chart shows the percentage of neurons with higher average responses in normal (black), cue-shift (blue), random cue presentation conditions (red), and both shifted and random laps (purple). 6 e) (Top) Dual cue location task: LED visual cue is given consistently at two positions on the track. Arrangement of cues for normal laps (left, $80 \%$ of 
laps), laps where first cue is omitted (middle, 10\%), and laps where the second cue is omitted (right, $10 \%$ ). (Bottom) Spatial firing rates of 433 significantly tuned cells on first day of task ( $N=3$ mice). $f$ ) Average spatial firing rates for significant cue cells (vs. cue omitted laps), for cells preferring cue position 1 (top) or cue position 2 (bottom) on first day of task (blue, $n=25$ pos $1, n=25$ pos2) and 4th day of task (pink, $\mathrm{n}=13$ pos $1, \mathrm{n}=20$ pos 2 cells). g) Spatial modulation index (rate non-preferred cue/preferred cue) for all cue cells on days $1-4$. ( $(p=0.0125$, day 1 vs. days 2,3 , or $4, n=50 / 40 / 50 / 33$, Wilcoxon rank sum test). $h$ ) Empirical cumulative probability distribution for all cue cells on days 1-4. See also Supplementary Figure 6.

a Population correlation

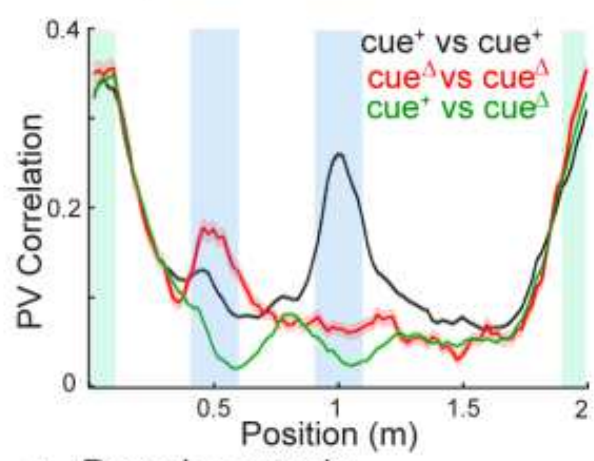

d Decoder error in normal and cue shift laps

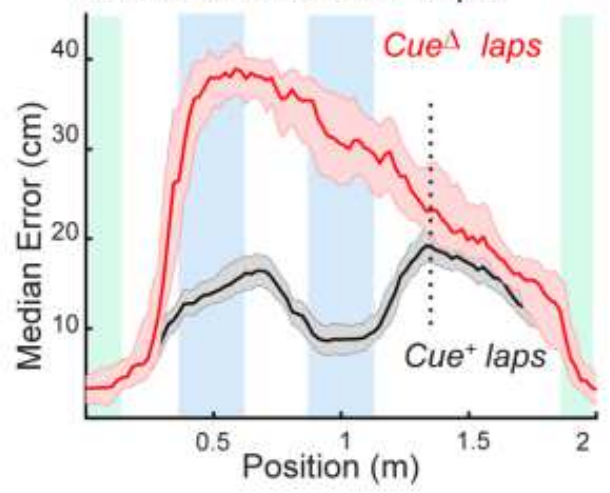

b

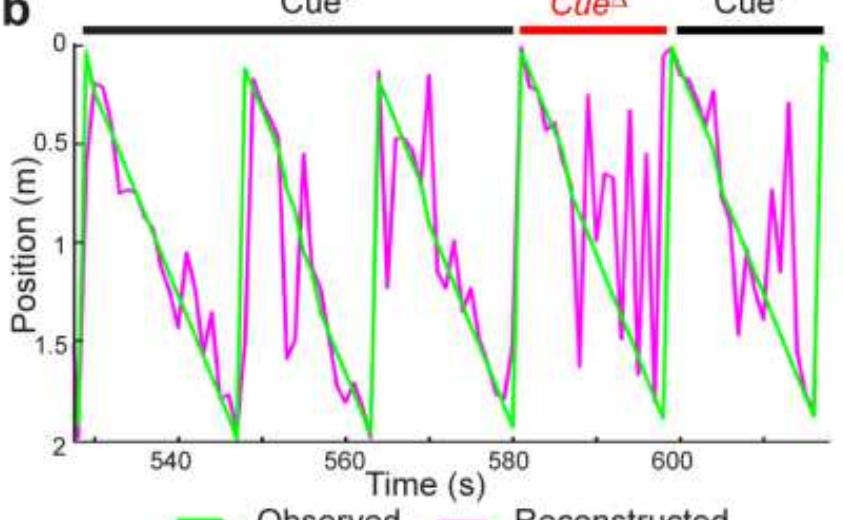

c Median Error

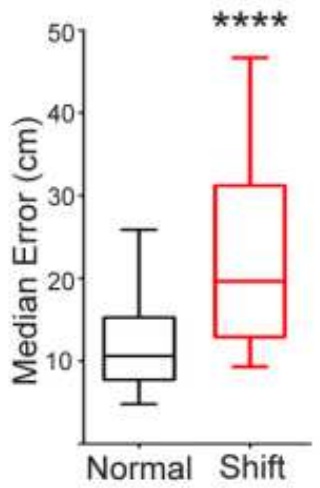

e Middle cue infleunced vs non middle cue influenced cells

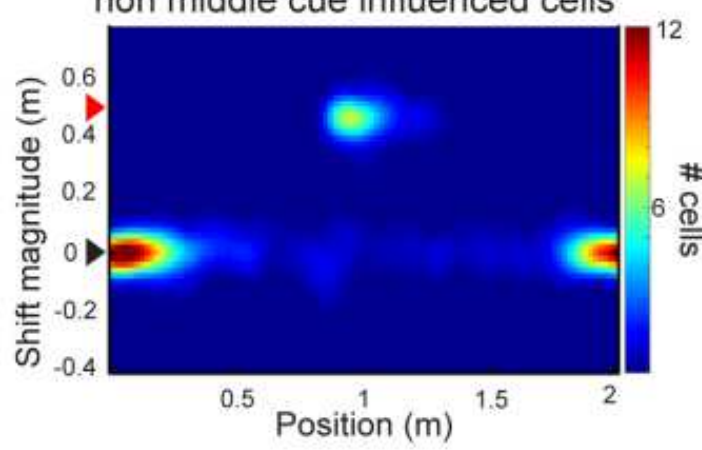

f

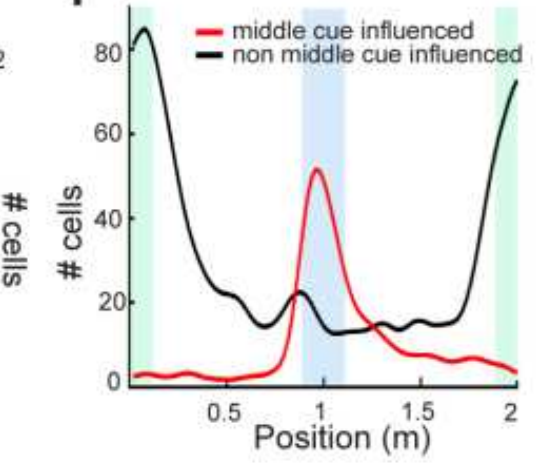

\section{Figure 4}

Cue shifts have limited effects on spatial encoding a) Population vector correlations for all spatially tuned cells for normal middle cue laps and shifted cue laps in cue shift task. Locations of the middle/shift and lap cue are shown blue and green shaded areas, respectively. b) Bayesian decoding of 4 normal and 1 cue shifted laps for a representative session, based upon activity from all spatially tuned granule cells. The magenta line shows predicted position at each bin, while the green line shows the observed position of the animal. c) Average decoding error for normal and cue shifted laps calculated as the absolute median distance between the decoded value in each time bin and the actual value of the position ( $p<0.0001$, Wilcoxon signed-rank test, $n=66$ sessions). Boxes, 25 th to 75 th percentiles; bars, median; whiskers, $99 \%$ range. d) Decoding error for each treadmill position in normal (black) and cue shifted laps (red). Shaded error region represents the difference of the median from the null distribution 
of median decoder error. Dotted line indicates point of statistical equality between normal and cue shifted laps. e) Distribution of cell firing location shift on shifted cue laps for all spatially tuned cells, plotted based upon normal tuning location $(n=66)$. Red arrowhead indicates cue shift distance, and thus marks cells that are directly influenced by the cue. Black arrowhead indicates cells with zero shift, and thus not influenced by the cue. f) Mean number of cells not influenced by the odor cue (shift magnitude $<0.05 \mathrm{~m}$ ) and cells shifting precisely along with the cue (shift magnitude $0.5 \mathrm{~m}+/-0.1 \mathrm{~m}$ ) at each track position (corresponding to cells in regions of black and red arrowheads in "b", respectively). Cue influenced cells are largely confined to locations around the cue itself, while non cue-influenced place cells are more evenly distributed, with a slight enrichment immediately preceding the middle cue. See also Supplementary Figure 7.
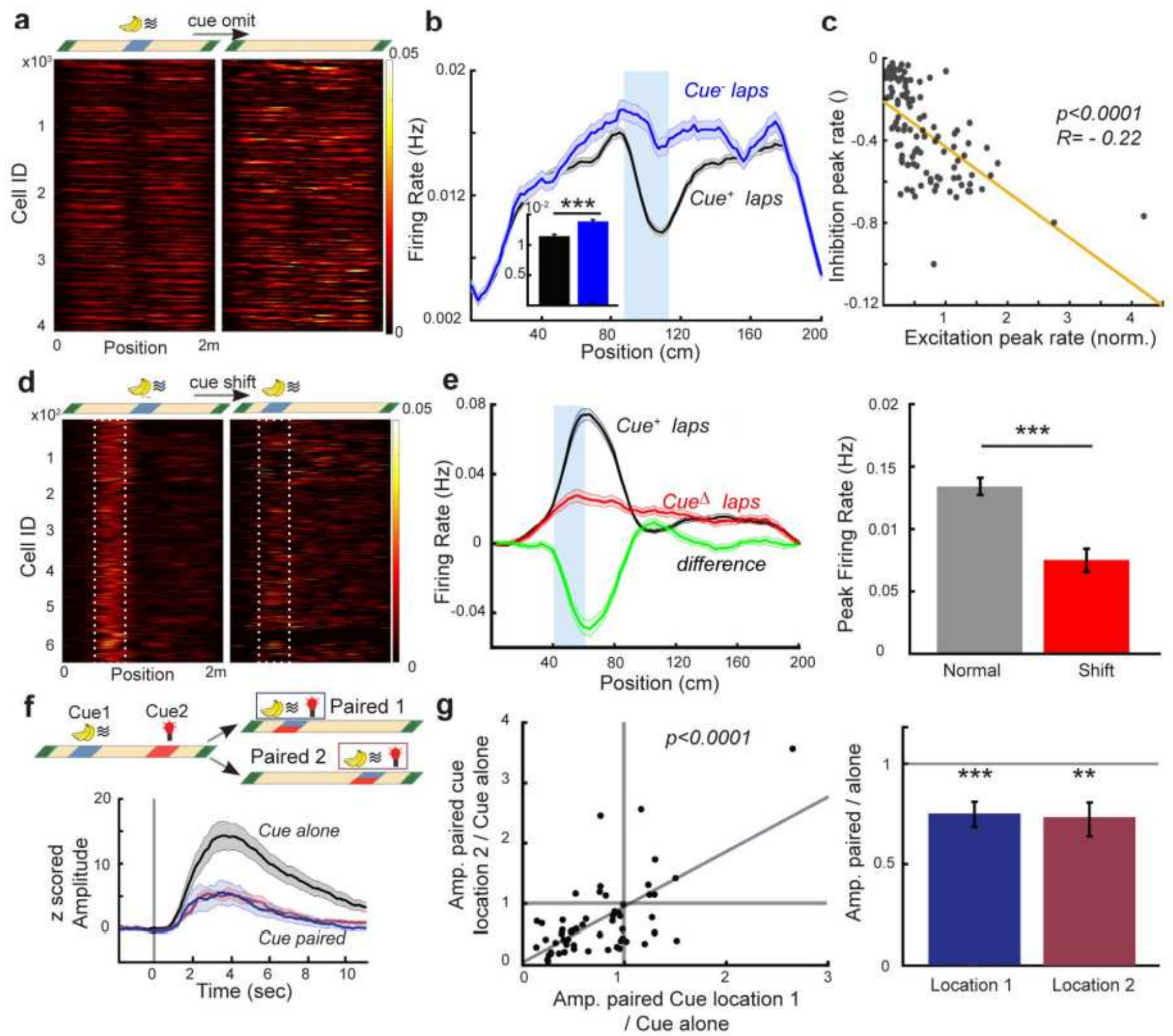

Figure 5 
Cue presentation leads to suppression of diverse types of DG responses a) Cue-related suppression of noisy, out-of-field firing. Spatial firing rates outside of the field center-of-mass $(+/-10 \mathrm{~cm})$ for significantly tuned cells during normal and cue omitted laps ( $n=4,091$ cells from 66 sessions). b) Average out-of-field firing rates from spatially tuned cells in the presence of the middle cue (black) compared to laps in which the cue is omitted (blue). Blue shaded area shows the cue delivery position. Inset, mean \pm SEM of firing rate, $p<0.001$, rank-sum test, $n=4,091$ cells. $c$ ) Comparison of the magnitude of cue-related excitation and inhibition. Session averaged in-field firing rate vs. out-of-field firing rate (66 sessions). Yellow, linear regression: $p<0.0001, R 2=-0.22$. d) Cue-related suppression of place cell firing. Spatial firing rates of place cells with firing fields at $40-80 \mathrm{~cm}$ during normal laps (left) and laps in which the cue is shifted to $40 \mathrm{~cm}$ (right). e) (Left) Average firing rate by position for the above place cells in normal laps when the cue is not presented at this location (Cue+, black), compared with laps in which the cue is shifted to this location (Cue $\Delta$, red; diff. in green). (Right), Average peak firing rate of the same cells in " $d$ " and "e", paired t-test, $p<0.001,645$ cells. $9 \mathrm{f}$ ) Cue-related suppression of cells responding to other cues. (Top) diagram of intermittent cue pairing experiment. Cues of two different modalities were presented at different locations, interspersed with paired presentation of these two cues at one of these locations. (Bottom) example cue-triggered averaged $\mathrm{Ca} 2+$ transients for a cue cell strongly active when the cue is presented alone (black) but with reduced responses when paired with a different cue, regardless of location (blue, pair location \#1, purple, location \#2). g) (Left) Amplitudes of paired cue responses at two pairing locations with respect to the response to the cue alone. Gray line represents the diagonal, $p<0.0001$, $\mathrm{R} 2=0.91, \mathrm{n}=56$ cells, 3 mice. (Right) Average relative response amplitude at the two pairing locations (Location 1: $0.75 \pm 0.061, \mathrm{p} 1=1.45 \times 10-4$; Location 2: $0.73 \pm 0.086, \mathrm{p} 2=0.003$, Wilcoxon Signed Rank Sum test, bar plots are mean \pm SEM). See also Supplementary Figure 8 .

\section{Supplementary Files}

This is a list of supplementary files associated with this preprint. Click to download.

- SupplementalFigures.pdf 